\title{
ALMOST SURE GLOBAL WELL POSEDNESS FOR THE RADIAL NONLINEAR SCHRÖDINGER EQUATION ON THE UNIT BALL II: THE 3D CASE
}

\author{
JEAN BOURGAIN AND AYNUR BULUT
}

\begin{abstract}
We extend the convergence method introduced in our works [8]10] for almost sure global well-posedness of Gibbs measure evolutions of the nonlinear Schrödinger (NLS) and nonlinear wave (NLW) equations on the unit ball in $\mathbb{R}^{d}$ to the case of the three dimensional NLS. This is the first probabilistic global well-posedness result for NLS with supercritical data on the unit ball in $\mathbb{R}^{3}$.

The initial data is taken as a Gaussian random process lying in the support of the Gibbs measure associated to the equation, and results are obtained almost surely with respect to this probability measure. The key tools used include a class of probabilistic a priori bounds for finite-dimensional projections of the equation and a delicate trilinear estimate on the nonlinearity, which when combined with the invariance of the Gibbs measure - enables the a priori bounds to be enhanced to obtain convergence of the sequence of approximate solutions.
\end{abstract}

\section{INTRODUCTION}

In the work at hand, we continue our study of Gibbs measure evolution for the nonlinear Schrödinger (NLS) and nonlinear wave (NLW) equations on the unit ball in Euclidean space, initiated in our earlier works [8]-[10]. In particular, the aim of the present article is to extend the almost sure global well-posedness result of [10], which was set on the unit ball in $\mathbb{R}^{2}$, to the setting of the unit ball in $\mathbb{R}^{3}$. The techniques involved are a further development of the method introduced in our work 9] for the nonlinear wave equation, combined with a delicate choice of function spaces adapted to the decay properties of the fundamental solution of the Schrödinger equation.

Date: June 10, 2018.

The research of J.B. was partially supported by NSF grants DMS-0808042 and DMS-0835373 and the research of A.B. was supported by NSF under agreement No. DMS-0808042 and the Fernholz Foundation. 
More precisely, we shall consider the initial value problem for the cubic NLS on the unit ball $B$ in $\mathbb{R}^{3}$,

$$
\left\{\begin{aligned}
i u_{t}+\Delta u-|u|^{2} u & =0 \\
\left.u\right|_{t=0} & =\phi,
\end{aligned}\right.
$$

where $u: I \times B \rightarrow \mathbb{C}$, subject to the Dirichlet boundary condition $\left.u\right|_{I \times \partial B}=0$, with randomly chosen radial initial data $\phi$; the sense in which the randomization is taken will be specified momentarily.

A fundamental property of (NLS) is that the equation takes the form of an infinite dimensional Hamiltonian system,

$$
i u_{t}=\frac{\partial H}{\partial \bar{u}}
$$

with conserved Hamiltonian

$$
H(\phi)=\frac{1}{2} \int_{B}|\nabla \phi|^{2}+\frac{1}{4} \int_{B}|\phi|^{4} .
$$

In the case that the spatial domain $B \subset \mathbb{R}^{3}$ is replaced with the $d$-dimensional torus $\mathbb{T}^{d}=\mathbb{R}^{d} / \mathbb{Z}^{d}$, a robust theory of almost sure global well posedness for the Cauchy problem was established in the seminal works [3]-[6] for a variety of general classes of nonlinearities including both the attractive and repulsive regimes; see also [7. for a brief survey of these results. The approach pioneered in this line of study was to obtain global control by exploiting the invariant properties of the Gibbs measure inherent in the Hamiltonian structure of the equation.

In preparation for our discussion below, we now outline the main steps of the approach pursued in those works:

(i) The first step is to consider a finite-dimensional projection of the Cauchy problem for (NLS), allowing access to an invariant Gibbs measure which gives global in time estimates for solutions.

(ii) A strong form of the local well-posedness theory driven by a contraction mapping principle then allows to show convergence of solutions for the finite-dimensional problems to a solution of the original equation. The key point in this step is to obtain estimates which are uniform in the projection parameter.

(iii) The two steps above are then combined to establish almost sure global wellposedness for the original Cauchy problem, (NLS) with no finite-dimensional projection.

(iv) The final step in the analysis is to establish the invariance of the limiting Gibbs measure with respect to the evolution given by the original, nonprojected, (NLS) equation.

We remark that the local theory in this approach is a consequence of fixed point arguments in suitable classes of function spaces. Although such results are 
usually available only for problems in which the initial data is subcritical or critical with respect to the scaling of the equation, nevertheless, the randomization gives additional integrability almost surely in the random variable, and can often enable the application to classes of supercritical data (see for instance [5] as well as [11]).

On the other hand, in the setting of the present paper a more substantial obstruction to implementing the approach described above is posed by the lack of robust Strichartz estimates on domains with boundary, which renders the fixed point technique ineffective for our purposes. Indeed, in the current work our arguments pursue a different path based on the treatment we introduced for the threedimensional nonlinear wave equation [9] and adapted to the two-dimensional NLS equation [10]. This approach is again based on a procedure of finite dimensional projection, with the goal of showing global well-posedness by establishing convergence for the sequence of solutions of the projected equations. However, with the fixed-point argument unavailable, the proof of convergence follows from a more delicate analysis of the fine behavior of solutions and their frequency interactions.

More precisely, the strategy in the present paper proceeds in the following steps:

(i') Construction of a suitable collection of function spaces used to establish convergence for the sequence of solutions to the finite-dimensional projections. Closely related to this is the identification of the relevant embeddings and basic interpolation properties of the spaces.

(ii') Establishing a priori bounds for solutions of the projected equations which remain uniform in the projection parameter.

(iii') The formulation of an estimate of the contribution of the nonlinearity. This estimate is the most delicate stage in the process, and serves to provide the decay necessary to establish convergence.

$\left(\mathrm{iv}^{\prime}\right)$ The above ingredients are then combined to establish convergence for the sequence of solutions of the projected equations, almost surely in the randomization. The limiting function is a solution of the original equation and is defined for arbitrarily long time intervals.

It is important to note that in our current setting the invariance of the Gibbs measure is an essential ingredient in obtaining the short-time local existence result, whereas in the fixed-point based approach of [3- 7 , the local theory is developed independently of the invariance of the Gibbs measure. This is a major distinction between the two approaches, and our use of the Gibbs measure at this stage of the argument can be seen as the key piece of probabilistic information which allows to overcome the lack of Strichartz estimates; for a complete discussion of this issue we refer the reader to our treatment in [9, where the technique was introduced.

Before giving the precise statement of our main results, we shall now describe the finite-dimensional projections which form the basis of our approach. 
1.1. Finite dimensional model and the Gibbs measure. We shall consider solutions to the truncated equation

$$
\left\{\begin{aligned}
i u_{t}+\Delta u-P_{N}\left(|u|^{2} u\right) & =0 \\
\left.u\right|_{t=0} & =P_{N} \phi,
\end{aligned}\right.
$$

where the operator $P_{N}$ is the projection to low frequencies defined by

$$
P_{N}\left(\sum_{n \in \mathbb{N}} a_{n} e_{n}(x)\right)=\sum_{n \leq N} a_{n} e_{n}(x) .
$$

with $\left(a_{n}\right) \in \ell^{2}$ and $\left(e_{n}\right)$ as the sequence of radial eigenfunctions of $-\Delta$ on $B$ with vanishing Dirichlet boundary conditions.

The initial value problem (1.1) is globally well-posed for every integer $N \geq 1$ : indeed, for any initial data $\phi \in L_{x}^{2}(B)$, there exists a unique global solution $u_{N}$ : $\mathbb{R} \times B \rightarrow \mathbb{C}$ satisfying the associated Duhamel formula,

$$
u_{N}(t)=e^{i t \Delta} P_{N} \phi+i \int_{0}^{t} e^{i(t-\tau) \Delta} P_{N}\left(\left|u_{N}\right|^{2} u_{N}\right)(\tau) d \tau .
$$

The Gibbs measure $\mu_{G}^{(N)}$ associated to (1.1) is defined (up to normalization factors) by

$$
\begin{aligned}
\mu_{G}^{(N)}(A) & =\int_{A} \exp \left(-H_{N}(\phi)\right) \prod_{i=1}^{N} d^{2} \phi \\
& =\int_{A} \exp \left(-\frac{1}{4}\left\|P_{N} \phi\right\|_{L_{x}^{4}}^{4}\right) d \mu_{F}^{(N)}(\phi), \quad A \in \mathcal{M}
\end{aligned}
$$

where

$$
H_{N}(\phi)=\frac{1}{2} \sum_{n \leq N} n^{2}|\widehat{\phi}(n)|^{2}+\frac{1}{4} \int_{B}\left|P_{N} \phi(x)\right|^{4} d x
$$

and $\mu_{F}^{(N)}$ is the free (Weiner) measure induced by the mapping

$$
\Omega \ni \omega \mapsto \phi_{\omega}:=\sum_{n \leq N} \frac{g_{n}(\omega)}{n \pi} e_{n},
$$

where $\left(g_{n}\right)$ is a sequence of IID normalized complex Gaussian random variables.

As we will see below, basic facts concerning the sequence of eigenfunctions $\left(e_{n}\right)$ ensure that the norms

$$
\|\phi\|_{H_{x}^{s}(B)}, \quad s<\frac{1}{2} \quad \text { and } \quad\left\|P_{N} \phi\right\|_{L_{x}^{p}(B)}, \quad p<6
$$

are finite $\mu_{F}^{(N)}$-almost surely for every $N \geq 1$. These facts dictate the spaces in which we look for solutions, and also serve to ensure that the measure $\mu_{G}^{(N)}$ is well-defined, nontrivial and normalizable. Finally, we remark that $\mu_{G}^{(N)}$ is invariant under the evolution of the truncated equation (1.1), that is to say

$$
\mu_{G}^{(N)}\left(\left\{\phi_{\omega}: \omega \in \Omega\right\}\right)=\mu_{G}^{(N)}\left(\left\{u_{N}(t): u_{N} \text { solves (1.1) with } \phi=\phi_{\omega}, \omega \in \Omega\right\}\right)
$$


for any $t \in \mathbb{R}$.

We are now ready to state the main result of this paper, which establishes almost sure convergence of the sequence of solutions to the truncated equation (1.1) as the truncation parameter $N$ tends to infinity.

Theorem. Let $(\Omega, p, \mathcal{M})$ be a given probability space. For each $N \in \mathbb{N}, \omega \in \Omega$ let $u_{N}$ denote the solution to (1.1) with initial data $P_{N} \phi=P_{N} \phi^{(\omega)}$. Then, almost surely in $\omega$, for every $s<1 / 2$ and $T<\infty$, there exists $u_{*} \in C_{t}\left([0, T) ; H_{x}^{s}(B)\right)$ such that $u_{N}$ converges to $u_{*}$ with respect to the norm $C_{t}\left([0, T) ; H_{x}^{s}(B)\right)$.

The proof of the theorem follows the approach described above, and can be roughly outlined as consisting of the following steps: (1) identification of the Fourier restriction spaces $X^{s, b}$ together with a variant $X_{|| \cdot|| \mid}$ as suitable classes of function spaces, (2) the derivation of a family of a priori bounds which are uniform in the finite-dimensional projection $P_{N},(3)$ a trilinear estimate on the nonlinearity which allows to enhance the a priori bounds into the decay necessary to establish convergence, and (4) a convergence argument for $N \rightarrow \infty$ which assembles the above ingredients.

The first step in the analysis is the choice of function spaces. As is by now familiar in the study of nonlinear dispersive equations, the spaces $X^{s, b}$ of [1, 2] are the natural spaces to carry out perturbation theory from the Duhamel formula (1.2). An additional component in the analysis in the present work is the need to consider short time intervals. To balance this requirement with the degenerating constant in the $X^{s, b}$-localization bound

$$
\|\psi f\|_{X^{s, b}} \lesssim \frac{1}{\delta^{b-1 / 2}}\|f\|_{X^{s, b}}, \quad b>\frac{1}{2}
$$

with $\psi(t)=\eta(t / \delta), \delta>0$, where $\eta: \mathbb{R} \rightarrow[0,1]$ is a smooth function such that $\eta=1$ on $[-1,1]$ and supp $\eta \subset[-2,2]$ (see, for instance, [7, Lecture 2]), we introduce also the slightly different space $X_{\||||| \mid}$for which the degenerating constant does not appear.

With the scale of function spaces identified, we next devote our attention to a priori bounds for solutions of the truncated equations (1.1), uniform in the truncation parameter. We first obtain such bounds in $L_{x}^{p} L_{t}^{q}$ norms, and subsequently extend the arguments to $X^{s, b}$ norms. To obtain the almost sure global well-posedness result of the theorem, it suffices to establish these bounds up to the exclusion of sets of small measure in the statistical ensemble. In view of this, the key observation is that by exploiting the invariance of the Gibbs measure, it is enough to establish analogous bounds for functions of the form

$$
\sum_{n \in \mathbb{N}} \frac{g_{n}(\omega)}{n \pi} e_{n}(x)
$$


This enables us to combine standard estimates for Gaussian processes and estimates on the eigenfunctions $e_{n}$ to obtain the desired bounds.

The next step is to obtain a trilinear estimate on the nonlinear term in the Duhamel formula. The argument to establish this bound proceeds by decomposing each of the three linear factors appearing in the nonlinearity $F(u)=|u|^{2} u$ into discrete frequencies and estimating the resulting frequency interactions. These estimates are performed using space-time norms, $X^{s, b}$-spaces and further probabilistic considerations based on the Gibbs measure invariance. In fact, we need to distinguish several frequency regions where different arguments apply. Introducing these regions requires certain care.

The final step in establishing the theorem is to assemble the above ingredients to show that the sequence $\left(u_{N}\right)$ of solutions to the truncated equations (1.1) is almost surely a Cauchy sequence in the space $C_{t}\left([0, T) ; H_{x}^{s}(B)\right)$. The core step in this argument takes the form of an estimate for the $X_{\||| \cdot||}$ norm of the difference $u_{N_{1}}-u_{N_{0}}$ for any integers $N_{1} \geq N_{0} \geq 1$. This bound is of the form

$$
||\left|u_{N_{1}}-u_{N_{0}}\right| \mid \lesssim N_{0}^{-c} \text { for some } c>0
$$

for all $\omega \in \Omega$ outside a singular set having small measure. The measures of these exceptional sets need to be sufficiently small in order to deduce an almost everywhere convergence result. Of course, large deviation estimates for Gaussian processes are essential here. The final stage of the argument consists in revisiting the probabilistic claims in order to justify the required quantitative form.

\section{Notation AND PRELIMINARIES}

Throughout our arguments we will frequently make use of a dyadic decomposition in frequency, writing

$$
f(x)=\sum_{n} \hat{f}(n) e_{n}(x)=\sum_{N \geq 1} \sum_{n \sim N} \hat{f}(n) e_{n}(x),
$$

where for each $n \in \mathbb{Z}$, the condition $n \sim N$ is characterized by $N \leq n \leq 2 N$.

For every $n \in \mathbb{N}$, define

$$
e_{n}(x)=\frac{\sin (n \pi|x|)}{|x|}
$$

and recall that $e_{n}$ is the $n$th radial eigenfunction of $-\Delta$ on $B$, with associated eigenvalue $n^{2}$. With this notation, we have the following estimates on the norms of the eigenfunctions:

$$
\left\|e_{n}\right\|_{L_{x}^{p}} \lesssim 1, \quad 1 \leq p<3 \quad \text { and } \quad\left\|e_{n}\right\|_{L_{x}^{p} \lesssim n^{1-\frac{3}{p}}, \quad p>3}
$$

along with the endpoint-type bound $\left\|e_{n}\right\|_{L_{x}^{3}} \lesssim(\log n)^{1 / 3}$. Moreover, the sequence $\left(e_{n}\right)$ also enjoys the following correlation bound:

$$
\left|c\left(n, n_{1}, n_{2}, n_{3}\right)\right| \lesssim \min \left\{n, n_{1}, n_{2}, n_{3}\right\},
$$


ALMOST SURE GLOBAL WELL POSEDNESS FOR RADIAL NLS ON THE 3D BALL 7

where we have set

$$
c\left(n, n_{1}, n_{2}, n_{3}\right)=\int_{B} e_{n}(x) e_{n_{1}}(x) e_{n_{2}}(x) e_{n_{3}}(x) d x .
$$

Another essential tool in our analysis is the following probabilistic estimate for sums of Gaussian random variables:

$$
\left\|\sum_{n} \alpha_{n} g_{n}(\omega)\right\|_{L^{q}(d \omega)} \lesssim \sqrt{q}\left(\sum_{n}\left|\alpha_{n}\right|^{2}\right)^{1 / 2}
$$

where $\left(\alpha_{n}\right) \in \ell^{2}, 2 \leq q<\infty$, and $\left(g_{n}\right)$ is a sequence of IID normalized complex Gaussians.

We also have the following multilinear version of the estimate (2.5):

$$
\left\|\sum_{n} \alpha_{n} h_{n}(\omega)\right\|_{L^{q}(d \omega)} \lesssim(\sqrt{q})^{k}\left\|\sum_{n} \alpha_{n} h_{n}(\omega)\right\|_{L^{2}(d \omega)}
$$

for every $k \geq 1,2 \leq q<\infty$ and each $h_{n}$ is a product of at most $k$ Gaussians taken from a sequence $\left(g_{n}\right)$ as above.

As a consequence, if $\left(g_{n}\right)$ is a sequence of normalized IID complex Gaussian random variables, the bound

$$
\left\|\sum_{n} \alpha_{n} \cdot\left(\left|g_{n}(\omega)\right|^{2}-1\right)\right\|_{L^{q}(d \omega)} \lesssim q\left(\sum_{n}\left|\alpha_{n}\right|^{2}\right)^{1 / 2} .
$$

holds for every $\left(\alpha_{n}\right) \in \ell^{2}$ and $1 \leq q<\infty$.

In the form (2.6), we note that the inequality remains valid in the vector-valued case, with $\left(\alpha_{n}\right)$ as elements of an arbitrary normed space $X$. See [12].

2.1. Description of the function spaces. Fix a time interval $I=[0, T)$ with $T>0$ sufficiently small, and let the space $X^{s, b}(I)$ denote the class of functions $f: I \times B \rightarrow \mathbb{C}$ representable as

$$
f(x, t)=\sum_{n, m} f_{n, m} e_{n}(x) e(m t), \quad(x, t) \in B \times I
$$

for which the norm

$$
\|f\|_{s, b}:=\left(\sum_{n, m}\langle n\rangle^{2 s}\left\langle n^{2}-m\right\rangle^{2 b}\left|f_{n, m}\right|^{2}\right)^{1 / 2}
$$

is finite, where the infinum is taken over all representations (2.8). We also refer the reader to the works [1]-[2], where these spaces were first introduced.

Moreover, when $f: I \times B \rightarrow \mathbb{C}$ has a representation (2.8), we shall define the function $T_{s, b} f$ via

$$
\left(T_{s, b} f\right)(x, t)=\sum_{n, m}\langle n\rangle^{s}\left\langle n^{2}-m\right\rangle^{b} f_{n, m} e_{n}(x) e(m t) .
$$

Our analysis requires to consider short time intervals $[0, T]$, where $T$ will depend on the truncation parameters. In order to establish contractive estimates for the 
nonlinear term, we need a variant of the $\|\cdot\|_{0, \frac{1}{2}}$-norm adapted to the time interval. We denote this norm by ||$|\cdot| \|_{0, \frac{1}{2} ; T}$, and its unit ball is generated by functions of the form

$$
\sum_{n, m} \frac{a_{n, m}}{\left(\left|n^{2}-m\right|+\frac{1}{T}\right)^{\frac{1}{2}}} e_{n}(x) e(m t)+\sum_{\substack{n, m \\\left|n^{2}-m\right|>\frac{1}{T}}} \frac{a_{n}}{\left|n^{2}-m\right|} e_{n}(x) e(m t)
$$

with

$$
\sum_{n, m}\left|a_{n, m}\right|^{2} \leq 1 \quad \text { and } \quad \sum_{n}\left|a_{n}\right|^{2} \leq 1
$$

Obviously, $\|\cdot\|_{0, b} \lesssim \||| \cdot|| \mid$ for $b<\frac{1}{2}$. One can similarly introduce norms $\left.\||\cdot|\|\right|_{s, \frac{1}{2} ; T}$ for $s>0$, but we will not need them for our purposes.

The next few lemmas put into evidence some basic properties of the norm $\||\cdot|\|$.

Lemma 2.1. Let ||$|f| \| \leq 1$. Then

$$
\frac{1}{T} \int_{0}^{T}\|f(t)\|_{L_{x}^{2}}^{2} d t<O(1)
$$

Proof. We first write $f$ as in (2.10). Then

$$
\begin{aligned}
\|f(t)\|_{L_{x}^{2}}^{2} & =\sum_{n}\left|\sum_{m} \frac{f_{n, m}}{\left(\left|n^{2}-m\right|+\frac{1}{T}\right)^{\frac{1}{2}}} e(m t)\right|^{2}+\sum_{n}\left|\sum_{\left|n^{2}-m\right|>\frac{1}{T}} \frac{e(m t)}{n^{2}-m}\right|^{2}\left|f_{n}\right|^{2} \\
& =(I)+(I I) .
\end{aligned}
$$

Taking $0 \leq \varphi \leq 2$ such that $\varphi \geq 1$ on $[0,1]$ and $\operatorname{supp} \hat{\varphi} \subset[-1,1]$, we have

$$
\begin{aligned}
\int_{0}^{T}(I) d t \leq \int(I) \varphi\left(\frac{t}{T}\right) d t & \leq T \sum_{n} \sum_{\substack{m, m^{\prime} \\
\left|m-m^{\prime}\right| \leq \frac{1}{T}}} \frac{\left|f_{n, m}\right|\left|f_{n, m^{\prime}}\right|}{\left|n^{2}-m\right|+\frac{1}{T}} \\
& \leq T^{2} \sum_{|k| \leq \frac{1}{T}} \sum_{n, m}\left|f_{n, m}\right|\left|f_{n, m+k}\right| \\
& \lesssim T\|f\|_{L_{t, x}^{2}}^{2} \\
& \lesssim T
\end{aligned}
$$

and similarly

$$
\begin{aligned}
\int_{0}^{T}(I I) d t & \lesssim T \sum_{n} \sum_{\substack{m, m^{\prime},\left|m-m^{\prime}\right| \leqslant \frac{1}{T} \\
\left|n^{2}-m\right|>\frac{1}{T},\left|n^{2}-m^{\prime}\right|>\frac{1}{T}}} \frac{\left|f_{n}\right|^{2}}{\left|n^{2}-m\right|\left|n^{2}-m^{\prime}\right|} \\
& \lesssim \sum_{\substack{n, m \\
\left|n^{2}-m\right|>\frac{1}{T}}} \frac{\left|f_{n}\right|^{2}}{\left|n^{2}-m\right|^{2}} \\
& \lesssim T
\end{aligned}
$$

The combination of these two bounds suffices to prove the claim. 
ALMOST SURE GLOBAL WELL POSEDNESS FOR RADIAL NLS ON THE 3D BALL 9

The next statement expresses an important duality property with respect to the Duhamel formula (1.2).

Lemma 2.2. Assume $f(x, t)=\sum_{n \in \mathbb{Z}_{+}, m \in \mathbb{Z}} f_{n, m} e_{n}(x) e(m t)$. Then

$$
\left\|\int_{0}^{t} e^{i(t-\tau) \Delta} f(\tau) d \tau\left|\| \lesssim \max _{\mid\|g\| \|_{0, \frac{1}{2} ; T \leq 1}}\right| \sum_{n, m} f_{n, m} g_{n, m} \mid\right.
$$

where $g(x, t)=\sum_{n \in \mathbb{Z}_{+}, m \in \mathbb{Z}} g_{n, m} e_{n}(x) e(m t)$.

Proof. Write

$$
\int_{0}^{t} e^{i(t-\tau) \Delta} f(\tau) d \tau=\sum_{n, m} f_{n, m} e_{n} \frac{e(m t)-e\left(n^{2} t\right)}{m-n^{2}}
$$

and decompose this as

$$
\begin{gathered}
\sum_{\left|m-n^{2}\right|>\frac{1}{T}} \frac{f_{n, m}}{m-n^{2}} e_{n} e(m t) \\
-\sum_{\left|m-n^{2}\right|>\frac{1}{T}} \frac{f_{n, m}}{m-n^{2}} e_{n} e\left(n^{2} t\right) \\
+\sum_{\left|m-n^{2}\right| \leq \frac{1}{T}} f_{n, m} e_{n} \frac{e(m t)-e\left(n^{2} t\right)}{m-n^{2}} .
\end{gathered}
$$

Hence we may write (2.15) as

$$
\sum_{\left|m-n^{2}\right|>\frac{1}{T}} \frac{b_{n, m}}{\left|m-n^{2}\right|^{\frac{1}{2}}} e_{n} e(m t)
$$

with

$$
b_{n, m}=\frac{ \pm f_{n, m}}{\left|m-n^{2}\right|^{\frac{1}{2}}}
$$

which satisfies

$$
\left(\sum_{n, m}\left|b_{n, m}\right|^{2}\right)^{\frac{1}{2}}=\left(\sum_{\left|m-n^{2}\right|>\frac{1}{T}} \frac{\left|f_{n, m}\right|^{2}}{\left|m-n^{2}\right|}\right)^{\frac{1}{2}}=\max \left|\sum_{\left|m-n^{2}\right|>\frac{1}{T}} f_{n, m} \frac{a_{n, m}}{\left|m-n^{2}\right|^{\frac{1}{2}}}\right|
$$

where the maximum is over sequences $\left(a_{n, m}\right)$ with

$$
\left(\sum_{n, m}\left|a_{n, m}\right|^{2}\right)^{1 / 2} \leq 1
$$

which takes care of the contribution of (2.15) to the left-hand side of (2.14).

Next, let

$$
\varphi(t)=\sum_{k} \hat{\varphi}(k) e(k t)
$$

satisfy $\varphi=1$ on $[0, T], \varphi \geq 0$ together with the condition $|\hat{\varphi}(k)| \lesssim \frac{T}{(1+|k| T)^{2}}$. 
For $0 \leq t \leq T$, write $(2.16)$ as

$$
\left[\sum_{n} b_{n} e_{n} e\left(n^{2} t\right)\right] \varphi(t)=\sum_{n, k} b_{n} e_{n} e\left(\left(n^{2}+k\right) t\right) \hat{\varphi}(k)
$$

with

$$
b_{n}=\sum_{\left|m-n^{2}\right|>\frac{1}{T}} \frac{f_{n, m}}{m-n^{2}}
$$

Thus (2.18) becomes

$$
\sum_{\left|m-n^{2}\right| \lesssim T} \frac{a_{n, m}}{\left|n^{2}-m\right|^{\frac{1}{2}}} e_{n} e(m t)
$$

with $a_{n, m}=b_{n}\left|n^{2}-m\right|^{\frac{1}{2}} \hat{\varphi}\left(n^{2}-m\right)$ and

$$
\begin{aligned}
\left(\sum_{n, m}\left|a_{n, m}\right|^{2}\right)^{\frac{1}{2}} & =\left(\sum_{n}\left|b_{n}\right|^{2} \sum_{k}|k||\hat{\varphi}(k)|^{2}\right)^{\frac{1}{2}} \\
& \lesssim\left(\sum_{n}\left|b_{n}\right|^{2}\right)^{\frac{1}{2}} \\
& =\max \left|\sum_{n, m} f_{n, m} \frac{a_{n}}{\left|m-n^{2}\right|} \chi_{\left|m-n^{2}\right|>\frac{1}{T}}\right| .
\end{aligned}
$$

with maximum taken over $\left(a_{n}\right)$ such that

$$
\sum_{n}\left|a_{n}\right|^{2} \leq 1
$$

which is the desired estimate for the contribution of (2.16).

Finally, for $0 \leq t \leq T$ and $\varphi$ as above, write (2.17) as

$$
\sum_{\left|m-n^{2}\right| \leq \frac{1}{T}} f_{n, m} e_{n} e\left(n^{2} t\right) \frac{e\left(\left(m-n^{2}\right) t\right)-1}{m-n^{2}} \varphi(t)
$$

and expand the exponential in a power series

$$
\sum_{s \geq 1} \frac{1}{s !}\left[\sum_{n} b_{n}^{(s)} e_{n} e\left(n^{2} t\right)\right]\left(\frac{t}{T}\right)^{s} \varphi(t)
$$

with

$$
b_{n}^{(s)}=\sum_{\left|m-n^{2}\right| \leq \frac{1}{T}} f_{n, m}\left(m-n^{2}\right)^{s-1} T^{s}
$$

to obtain

$$
\left(\sum_{n}\left|b_{n}^{(s)}\right|^{2}\right)^{\frac{1}{2}} \leq T\left[\sum_{n}\left(\sum_{\left|m-n^{2}\right| \leq \frac{1}{T}}\left|f_{n, m}\right|\right)^{2}\right]^{\frac{1}{2}} \leq \sqrt{T}\left(\sum_{\left|m-n^{2}\right| \leq \frac{1}{T}}\left|f_{n, m}\right|^{2}\right)^{\frac{1}{2}} .
$$

For each $s$, let $\psi_{s}(t)=\sum_{k} \hat{\psi}_{s} e(k t)$ be an extension of $\left(\frac{t}{T}\right)^{s}, 0 \leq t \leq T$ such that

$$
\left|\psi_{s}\right| \leq 2 \text { and }\left|\psi_{s}^{\prime}\right| \leq 10 s T^{-1} \text {. }
$$

Then

$$
\left|\widehat{\varphi \psi_{s}}(k)\right| \leq\left\|\varphi \psi_{s}\right\|_{L_{x}^{1}} \leq 2\|\varphi\|_{L_{x}^{1}} \lesssim T
$$


and

$$
\left\|\varphi \psi_{s}\right\|_{H^{\frac{1}{2}}} \lesssim\left\|\varphi \psi_{s}\right\|_{L_{x}^{2}}^{\frac{1}{2}}\left(\left\|\varphi^{\prime} \psi_{s}\right\|_{L_{x}^{2}}+\left\|\varphi \psi_{s}^{\prime}\right\|_{L_{x}^{2}}\right)^{\frac{1}{2}} \lesssim T^{\frac{1}{4}}\left(T^{-\frac{1}{2}}+s T^{-\frac{1}{2}}\right)^{\frac{1}{2}} \lesssim s^{\frac{1}{2}},
$$

which in view of (2.19) gives the desired representation of (2.17).

The norm ||$|\cdot|||$ does not quite control the $L_{0 \leq t \leq T}^{\infty} L_{x}^{2}$-norm. However, the following holds, which will suffice for our purpose.

Lemma 2.3. Let $f$ and $g$ have expansions as in Lemma 2.2. Then

$$
\left|\sum_{n, m} f_{n, m} g_{n, m}\right| \lesssim T|||f|\|\cdot|\|g \mid\| \cdot
$$

Proof. From the representation (2.10), we obtain

$$
\begin{aligned}
\sum_{n, m}\left|f_{n, m}\right|\left|g_{n, m}\right| \lesssim \sum_{n, m} & \frac{a_{n, m} b_{n, m}}{\left|n^{2}-m\right|+\frac{1}{T}}+\sum_{\left|n^{2}-m\right|>\frac{1}{T}} \frac{a_{n} b_{n, m}}{\left|n^{2}-m\right|^{3 / 2}} \\
& +\sum_{\left|n^{2}-m\right|>\frac{1}{T}} \frac{a_{n, m} b_{n}}{\left|n^{2}-m\right|^{3 / 2}}+\sum_{\left|n^{2}-m\right|>\frac{1}{T}} \frac{a_{n} b_{n}}{\left|n^{2}-m\right|^{2}}
\end{aligned}
$$

with

and

$$
\sum_{n, m}\left|a_{n, m}\right|^{2} \leq 1, \quad \sum_{n, m}\left|b_{n, m}\right|^{2} \leq 1, \quad \sum_{n}\left|a_{n}\right|^{2} \leq 1
$$

$$
\sum_{n}\left|b_{n}\right|^{2} \leq 1
$$

By the Cauchy-Schwarz inequality, the first term is bounded by $T$, while the second term is bounded by

$$
\left\{\sum_{n}\left(\sum_{\left\{m:\left|n^{2}-m\right|>\frac{1}{T}\right\}} \frac{\left|b_{n, m}\right|}{\left|n^{2}-m\right|^{3 / 2}}\right)^{2}\right\}^{\frac{1}{2}} \lesssim T .
$$

The estimate for the third term is similar. Estimating the last term, we obtain the bound

$$
T \sum_{n} a_{n} b_{n} \lesssim T
$$

which allows to complete the lemma.

Next, we establish several inequalities bounding suitable $L_{x}^{p} L_{t}^{q}$-norms in terms of $X^{s, b}$-norms. These will be essential to our analysis.

Lemma 2.4. The spaces $X^{s, b}$ obey the following embedding relations:

(i) For $2<p<3$ and $b_{1}>\frac{1}{4}$,

$$
\|f\|_{L_{x}^{p} L_{t}^{2}} \lesssim\|f\|_{0, b_{1}} \text {. }
$$

(ii) For $3<p<6, s>1-\frac{3}{p}$ and $b_{2}>\frac{1}{2}$,

$$
\|f\|_{L_{x}^{p} L_{t}^{4}} \lesssim\|f\|_{s, b_{2}}
$$


(iii) For $\frac{1}{4}<b_{3}<\frac{1}{2}$ and $\epsilon>0$,

$$
\|f\|_{L_{x}^{3} L_{t}^{\frac{4}{3-4 b_{3}}}} \lesssim\|f\|_{\epsilon, b_{3}} .
$$

(iv) For $b_{4}>\frac{1}{2}$ and $s>\frac{1}{2}$,

$$
\|f\|_{L_{x}^{3} L_{t}^{\infty}} \lesssim\|f\|_{s, b_{4}} .
$$

(v) For $3 \leq p \leq 6,4 \leq q \leq \infty, s>\frac{3}{2}-\frac{3}{p}-\frac{2}{q}$ and $b_{5}>\frac{1}{2}$

$$
\|f\|_{L_{x}^{p} L_{t}^{q}} \lesssim\|f\|_{s, b_{5}} .
$$

(vi) For $\frac{1}{4}<b_{6}<\frac{1}{2}, 3<p<\frac{6}{3-4 b_{6}}, \frac{4}{3-4 b_{6}}<q<\infty$, and $s>\frac{5}{2}-\frac{3}{p}-\frac{2}{q}-2 b_{6}$,

$$
\|f\|_{L_{x}^{p} L_{t}^{q}} \lesssim\|f\|_{s, b_{6}} .
$$

(vii) For $2 \leq p<\frac{8}{3}$ and $b_{7}>\frac{1}{2}$,

$$
\|f\|_{L_{x}^{p} L_{t}^{p}} \lesssim\|f\|_{0, b_{7}}
$$

(viii) For $\frac{1}{4}<b_{8}<\frac{1}{2}, p<\frac{24}{4 b_{8}+7}$, and $q<\frac{8}{5-4 b_{8}}$,

$$
\|f\|_{L_{x}^{p} L_{t}^{q}} \lesssim\|f\|_{0, b_{8}}
$$

Proof. We begin with (i). Let $2<p<3$ be given. Then for every $f$ as in (2.8), applying the Plancherel identity in time followed by the Minkowski inequality, the eigenfunction estimate (2.4) and the Cauchy-Schwarz inequality, we have

$$
\begin{aligned}
\|f\|_{L_{x}^{p} L_{t}^{2}} & \lesssim\left(\sum_{m}\left\|\sum_{n} f_{m, n} e_{n}(x)\right\|_{L_{x}^{p}}^{2}\right)^{1 / 2} \\
& \lesssim\left(\sum_{m}\left(\sum_{n}\left|f_{m, n}\right|\right)^{2}\right)^{1 / 2} \\
& \lesssim\left(\sum_{m}\left(\sum_{n}\left\langle m-n^{2}\right\rangle^{2 b}\left|f_{m, n}\right|^{2}\right)\left(\sum_{n} \frac{1}{\left\langle m-n^{2}\right\rangle^{2 b}}\right)\right)^{1 / 2} .
\end{aligned}
$$

Observing that $b>\frac{1}{4}$ implies

$$
\sup _{m} \sum_{n} \frac{1}{\left\langle m-n^{2}\right\rangle^{2 b}}<\infty
$$

then establishes (i) as desired.

We now turn to (ii), for which we argue as in the proof of [10, Lemma 2.2]. Let $3<p<6$ be given. Then, writing (2.8) in the form

$$
f(t, x)=\sum_{m}\left(\sum_{n} f_{m+n^{2}, n} e_{n}(x) e\left(n^{2} t\right)\right) e(m t),
$$


we perform a dyadic decomposition into intervals $m \sim M, n \sim N$, expand the square inside the norm $\left\||\cdot|^{2}\right\|_{L_{x}^{p / 2} L_{t}^{2}}^{1 / 2}$, and use the Plancherel identity in the $t$ variable to obtain

$$
\begin{aligned}
\|f\|_{L_{x}^{p} L_{t}^{4}} & \lesssim \sum_{M, N} \sum_{m \sim M}\left\|\sum_{n \sim N} f_{m+n^{2}, n} e_{n}(x) e\left(n^{2} t\right)\right\|_{L_{x}^{p} L_{t}^{4}} \\
& \lesssim \sum_{M, N} \sum_{m \sim M}\left\|\left(\sum_{\ell}\left|\sum_{\substack{n, n^{\prime} \sim N \\
n^{2}+\left(n^{\prime}\right)^{2}=\ell}} f_{m+n^{2}, n} f_{m+\left(n^{\prime}\right)^{2}, n^{\prime}} e_{n}(x) e_{n^{\prime}}(x)\right|^{2}\right)^{1 / 2}\right\|_{L_{x}^{p / 2}}^{1 / 2} \\
& \lesssim \sum_{M, N} \sum_{m \sim M}\left(\sup _{\ell} \sum_{\substack{n, n^{\prime} \sim N \\
n^{2}+\left(n^{\prime}\right)^{2}=\ell}} 1\right)^{1 / 4}\left\|\left(\sum_{n \sim N}\left|f_{m+n^{2}, n}\right|^{2} e_{n}(x)^{2}\right)\right\|_{L_{x}^{p / 2}}^{1 / 2} .
\end{aligned}
$$

where we have used the Cauchy-Schwarz inequality to obtain the last bound.

Note that arithmetic considerations associated with lattice points on circles (see, for instance [10, Lemma 2.1] and the comments in the proof of [10, Lemma 2.2]) entail the bound

$$
\sup _{\ell \geq 0}\left|\left\{\left(n, n^{\prime}\right) \in[0, N]^{2}: n^{2}+\left(n^{\prime}\right)^{2}=\ell\right\}\right| \lesssim N^{\epsilon}
$$

for any $\epsilon>0$ (where the implicit constant may depend on $\epsilon$ ).

Set $\epsilon=s-\left(1-\frac{3}{p}\right)$. Then, using (2.21) followed by the Minkowski inequality, the eigenfunction estimates (2.21), and the Cauchy-Schwarz inequality in the summation over $m \sim M$,

$$
\begin{aligned}
(2.20) & \lesssim \sum_{M, N} \sum_{m \sim M} N^{\epsilon / 4}\left(\sum_{n \sim N}\left|f_{m+n^{2}, n}\right|^{2}\left\|e_{n}(x)\right\|_{L_{x}^{p}}^{2}\right)^{1 / 2} \\
& \lesssim \sum_{M, N} \sum_{m \sim M} N^{\epsilon / 4}\left(\sum_{n \sim N} n^{2-\frac{6}{p}}\left|f_{m+n^{2}, n}\right|^{2}\right)^{1 / 2} \\
& \lesssim \sum_{M, N} N^{\epsilon / 4} M^{\frac{1}{2}}\left(\sum_{m \sim M} \sum_{n \sim N} n^{2-\frac{6}{p}}\left|f_{m+n^{2}, n}\right|^{2}\right)^{1 / 2} \\
& \lesssim \sum_{M, N} N^{-3 \epsilon / 4} M^{\frac{1}{2}-b}\left(\sum_{\substack{n \sim N \\
m-n^{2} \sim M}}\langle n\rangle^{2 s}\left\langle m-n^{2}\right\rangle^{2 b}\left|f_{m, n}\right|^{2}\right)^{1 / 2} \\
& \lesssim\|f\|_{s, b},
\end{aligned}
$$

since

$$
\sum_{M, N} N^{-3 \epsilon / 2} M^{1-2 b}<\infty
$$

This completes the proof of part (ii) of the lemma.

The inequality stated in part (iii) now follows from parts (i) and (ii) by standard interpolation arguments. 
Next, we prove (iv). Since $b_{4}>\frac{1}{2}$, it suffices to consider $f$ of the form

$$
f(x, t)=\sum_{n} a_{n} e_{n}(x) e\left(n^{2} t\right)
$$

with

$$
\sum_{n} n^{2 s}\left|a_{n}\right|^{2} \leq 1
$$

It follows from the Cauchy-Schwarz inequality that for any $\epsilon>0$

$$
|f(x, t)| \lesssim\left[\sum_{n}\left|a_{n}\right|^{2} n^{1+\epsilon}\left|e_{n}(x)\right|^{2}\right]^{\frac{1}{2}}
$$

and hence

$$
\|f\|_{L_{x}^{3} L_{t}^{\infty}} \lesssim\left[\sum_{n}\left|a_{n}\right|^{2} n^{1+\epsilon}\left\|e_{n}\right\|_{L_{x}^{3}}^{2}\right]^{\frac{1}{2}}<O(1)
$$

Inequality (v) then follows by interpolation between (ii) and (iv), while (vi) is obtained by interpolating between (i) and (v).

We prove (vii), taking $f$ of the form

$$
f(x, t)=\sum_{n} a_{n} e_{n}(x) e\left(n^{2} t\right)=\sum_{n} a_{n} \frac{\sin \pi n r}{r} e\left(n^{2} t\right)
$$

with $r=|x|$ and $\sum_{n}\left|a_{n}\right|^{2} \leq 1$.

Fix $0<\rho \leq 1$ and consider values of $x$ in the annulus $\frac{\rho}{2} \leq r \leq \rho$. We make two estimates. We first note that

$$
\begin{aligned}
\|f\|_{L_{|x| \sim \rho}^{4} L_{t}^{4}} & \leq \frac{1}{\sqrt{\rho}}\left\|\sum_{n} a_{n}(\sin \pi n r) e\left(n^{2} t\right)\right\|_{L_{r \leq 1}^{4} L_{|t| \leq 1}^{4}} \\
& \leq \frac{1}{\sqrt{\rho}}\left[\max _{k, \ell}\left|\left\{\left(n, n^{\prime}\right) \in \mathbb{Z}^{2} ; n \pm n^{\prime}=k, n^{2}+\left(n^{\prime}\right)^{2}=\ell\right\}\right|\right]^{\frac{1}{4}} \\
& \lesssim \frac{1}{\sqrt{\rho}} .
\end{aligned}
$$

On the other hand, one has

$$
\|f\|_{L_{|x| \sim \rho}^{2} L_{t}^{2}} \leq\left\|\sum_{n} a_{n}(\sin \pi n r) e\left(n^{2} t\right)\right\|_{L_{r \sim \rho}^{2} L_{|t|<1}^{2}} \lesssim \sqrt{\rho} .
$$

Hence (vii) follows by interpolation between (2.22), (2.23) and summation over dyadic $\rho=2^{-j}$.

Finally, (viii) is obtained by interpolation between (i) and (vii). This completes the proof of Lemma 2.4. 


\section{A PRIORI UNIFORM BOUNDS}

In this section, we establish $X^{s, b}$ bounds on solutions of the truncated equation (1.1) which are uniform in the truncation parameter $N$. For this purpose, we will first obtain a preliminary uniform estimate on the norms $L_{x}^{p} L_{t}^{q}$ for suitable values of $p$ and $q$. In particular, we have the following:

Lemma 3.1. For every $0 \leq s<1 / 2,1 \leq p<\frac{6}{1+2 s}, 1 \leq q<\infty$, there exists a constant $C>0$ such that for every $N>0$ one has the bound

$$
\mu_{F}^{(N)}\left(\left\{\phi:\left\|(\sqrt{-\Delta})^{s} u\right\|_{L_{x}^{p} L_{t}^{q}}>\lambda\right\}\right) \lesssim \exp \left(-c \lambda^{c}\right),
$$

where $u=u_{N}$ is a solution to the truncated equation (1.1) associated to initial data $\phi$ (truncated as $\left.P_{N} \phi\right)$.

Proof. Without loss of generality we may assume $p>3$. It suffices to show that (3.1) holds with $\mu_{F}^{(N)}$ replaced by the Gibbs measure $\mu_{G}$. Indeed, suppose that one has

$$
\mu_{G}\left(A_{\lambda}\right) \leq C \exp \left(-c \lambda^{c}\right)
$$

with

$$
A_{\lambda}:=\left\{\phi:\left\|(\sqrt{-\Delta})^{s} u\right\|_{L_{x}^{p} L_{t}^{q}}>\lambda\right\}, \quad \lambda>0 .
$$

Then, fixing $\lambda_{1}>0$, we have

$$
\begin{aligned}
\mu_{F}^{(N)}\left(A_{\lambda}\right) & =\mu_{F}^{(N)}\left(A_{\lambda} \cap\left\{\phi:\|\phi\|_{L_{x}^{4}}>\lambda_{1}\right\}\right)+\mu_{F}^{(N)}\left(A_{\lambda} \cap\left\{\phi:\|\phi\|_{L_{x}^{4}} \leq \lambda_{1}\right\}\right) \\
& \lesssim \mu_{F}^{(N)}\left(\left\{\phi:\|\phi\|_{L_{x}^{4}}>\lambda_{1}\right\}\right)+\exp \left(\frac{1}{4} \lambda_{1}^{4}\right) \mu_{G}\left(A_{\lambda}\right) \\
& \lesssim \mu_{F}^{(N)}\left(\left\{\phi:\|\phi\|_{L_{x}^{4}}>\lambda_{1}\right\}\right)+\exp \left(\frac{1}{4} \lambda_{1}^{4}\right) \exp \left(-c \lambda^{c}\right) .
\end{aligned}
$$

To estimate the first term in (3.3), we fix $q_{1} \geq 4$ and appeal to the Tchebyshev and Minkowski inequalities followed by the estimate (2.5) on sums of Gaussian random variables. This gives

$$
\begin{aligned}
\mu_{F}^{(N)}\left(\left\{\phi:\|\phi\|_{L_{x}^{4}}>\lambda_{1}\right\}\right) & \lesssim \frac{1}{\lambda_{1}^{q_{1}}}\left[\mathbb{E}_{\mu_{F}^{(N)}}\|\phi\|_{L_{x}^{4}}^{q_{1}}\right] \\
& \leq \frac{1}{\lambda_{1}^{q_{1}}}\left\|\left(\mathbb{E}_{\mu_{F}^{(N)}}\left[\left(\sum_{n} \frac{g_{n}(\omega)}{n} e_{n}(x)\right)^{q_{1}}\right]\right)^{1 / q_{1}}\right\|_{L_{x}^{4}}^{q_{1}} \\
& \lesssim\left(\frac{\sqrt{q_{1}}}{\lambda_{1}}\right)^{q_{1}}\left\|\left(\sum_{n} \frac{\left|e_{n}(x)\right|^{2}}{n^{2}}\right)^{1 / 2}\right\|_{L_{x}^{4}}^{q_{1}} \\
& \lesssim\left(\frac{\sqrt{q_{1}}}{\lambda_{1}}\right)^{q_{1}}\left(\sum_{n} \frac{\left\|e_{n}\right\|_{L_{x}^{4}}^{2}}{n^{2}}\right)^{q_{1} / 2} .
\end{aligned}
$$

where in obtaining the last inequality we have used the Minkowski inequality. 
Invoking now the eigenfunction estimate (2.2),

$$
(\underline{3.4}) \lesssim\left(\frac{\sqrt{q_{1}}}{\lambda_{1}}\right)^{q_{1}}\left(\sum_{n} n^{-3 / 2}\right)^{q_{1} / 2} \lesssim\left(\frac{\sqrt{q_{1}}}{\lambda_{1}}\right)^{q_{1}}
$$

We therefore obtain

$$
\mu_{F}^{(N)}\left(A_{\lambda}\right) \lesssim\left(\frac{\sqrt{q_{1}}}{\lambda_{1}}\right)^{q_{1}}+\exp \left(\frac{1}{4} \lambda_{1}^{4}\right) \mu_{G}(A),
$$

so that optimizing in the choice of $q_{1}$ gives

$$
\mu_{F}^{(N)}\left(A_{\lambda}\right) \lesssim \exp \left(-c \lambda_{1}^{c}\right)
$$

as desired.

It therefore suffices to show (3.2), which we recall was the desired inequality with the measure $\mu_{F}^{(N)}$ replaced by the (invariant) Gibbs measure $\mu_{G}=\mu_{G}^{(N)}$. We argue as above: fixing $q_{2} \geq \max \{p, q\}$ and invoking the Tchebychev and Minkowski inequalities, one has

$$
\begin{aligned}
\mu_{G}\left(A_{\lambda}\right) & \leq \lambda^{-q_{2}} \mathbb{E}_{\mu_{G}}\left[\left\|(\sqrt{-\Delta})^{s} u\right\|_{L_{x}^{p} L_{t}^{q}}^{q_{2}}\right] \\
& \lesssim \lambda^{-q_{2}}\left\|\left(\mathbb{E}_{\mu_{G}}\left[\left((\sqrt{-\Delta})^{s} u\right)^{q_{2}}\right]\right)^{1 / q_{2}}\right\|_{L_{x}^{p} L_{t}^{q}}^{q_{2}}
\end{aligned}
$$

Now, using the invariance of the Gibbs measure $\mu_{G}=\mu_{G}^{(N)}$ with respect to the truncated evolution (with $u=u_{N}$ being a solution of the truncated equation) followed by the estimate for sums of Gaussian random variables given by (2.5),

$$
\begin{aligned}
(3.5) & \lesssim \lambda^{-q_{2}}\left\|\left(\mathbb{E}_{\mu_{G}}\left[\left(\sum_{n} \frac{g_{n}(\omega)}{n^{1-s}} e_{n}\right)^{q_{2}}\right]\right)^{1 / q_{2}}\right\|_{L_{x}^{p} L_{t}^{q}}^{q_{2}} \\
& \lesssim\left(\frac{\sqrt{q_{2}}}{\lambda}\right)^{q_{2}}\left\|\sum_{n} \frac{\left|e_{n}(x)\right|^{2}}{n^{2(1-s)}}\right\|_{L_{x}^{p / 2}}^{q_{2} / 2}
\end{aligned}
$$

To conclude, we use the eigenfunction estimate (2.2) together with the condition $p<\frac{6}{1+2 s}$ to get the bound

$$
\begin{aligned}
\left\|\sum_{n} \frac{\left|e_{n}(x)\right|^{2}}{n^{2(1-s)}}\right\|_{L_{x}^{p / 2}}^{q_{2} / 2} & \lesssim\left(\sum_{n} \frac{1}{n^{2(1-s)}}\left\|e_{n}(x)\right\|_{L_{x}^{p}}^{2}\right)^{q_{2} / 2} \\
& \lesssim\left(\sum_{n} n^{2\left(s-\frac{3}{p}\right)}\right)^{q_{2} / 2} \\
& \lesssim 1
\end{aligned}
$$

Hence

$$
\mu_{G}\left(A_{\lambda}\right) \lesssim\left(\frac{\sqrt{q_{2}}}{\lambda}\right)^{q_{2}}
$$

Optimizing the choice of $q_{2}$ in (3.6) as for $q_{1}$ above gives

$$
\mu_{F}^{(N)}\left(\left\{\phi:\left\|(\sqrt{-\Delta})^{s} u\right\|_{L_{x}^{p} L_{t}^{q}}>\lambda\right\}\right) \lesssim \exp \left(-c \lambda^{2}\right)
$$

as desired. 
We are now ready to establish uniform $X^{s, b}$-bounds.

Proposition 3.2. Fix $0 \leq s<\frac{1}{2}$ and $\frac{1}{2}<b<\frac{3}{4}$. Then there exists $C>0$ such that for all $N>0$, if $u=u_{N}$ is a solution to the truncated equation (1.1), then

$$
\mu_{F}^{(N)}\left(\left\{\phi:\|u\|_{s, b}>\lambda\right\}\right) \lesssim \exp \left(-c_{1} \lambda^{c_{2}}\right)
$$

Proof. Let $s \in\left[0, \frac{1}{2}\right)$ and $b \in\left(\frac{1}{2}, \frac{3}{4}\right)$ be given. Fix $N \geq 1$ and write the Duhamel formula

$$
u(t)=e^{i t \Delta} \phi+\int_{0}^{t} e^{i(t-\tau) \Delta}|u|^{2} u(\tau) d \tau .
$$

We estimate both the linear and nonlinear terms in (3.7) individually. We begin with the linear term. Let $T_{s, b}$ be the operator defined in (2.9). Then, fixing $q \geq 2$ and invoking the Tchebychev and Minkowski inequalities, one has

$$
\begin{aligned}
\mu_{F}^{(N)}\left(\left\{\phi:\left\|e^{i t \Delta} \phi\right\|_{s, b}>\lambda\right\}\right) & \leq \lambda^{-q} \mathbb{E}_{\omega}\left[\left\|T_{s, b} e^{i t \Delta} \phi\right\|_{L_{t, x}^{2}}^{q}\right] \\
& \lesssim \lambda^{-q}\left\|\mathbb{E}_{\omega}\left[\left(T_{s, b} e^{i t \Delta} \phi\right)^{q}\right]^{1 / q}\right\|_{L_{t, x}^{2}}^{q} \\
& \lesssim \lambda^{-q} q^{q / 2}\left\|\sum_{n} \frac{\left|e_{n}(x)\right|^{2}}{n^{2(1-s)}}\right\|_{L_{t, x}^{1}}^{q / 2} \\
& \lesssim \lambda^{-q} q^{q / 2}
\end{aligned}
$$

Appropriate choice of $\lambda$ gives

$$
\mu_{F}^{(N)}\left(\left\{\phi:\left\|e^{i t \Delta} \phi\right\|_{s, b}>\lambda\right\}\right) \lesssim \exp \left(-c \lambda^{2}\right) .
$$

Turning to the integral term, we set $f=|u(\tau)|^{2} u(\tau)$ and observe that the expansion $f(x, \tau)=\sum_{m, n} f_{n, m} e_{n}(x) e(m \tau)$ leads to

$$
\begin{aligned}
\int_{0}^{t} e^{i(t-\tau) \Delta} f(\tau) d \tau & =\int_{0}^{t}\left(\sum_{m, n} f_{n, m} e_{n}(x) e\left((t-\tau) n^{2}+m \tau\right)\right) d \tau \\
& =\sum_{m, n} \frac{i f_{n, m}}{\left(n^{2}-m\right)} e_{n}(x)\left(e\left(t n^{2}\right)-e(t m)\right) .
\end{aligned}
$$

Applying Hölder's inequality and recalling $b>\frac{1}{2}$, we obtain

$$
\begin{aligned}
\left\|\int_{0}^{t} e^{i(t-\tau) \Delta} f(\tau) d \tau\right\|_{s, b} & \lesssim\left(\sum_{n, m} \frac{\langle n\rangle^{2 s}\left|f_{n, m}\right|^{2}}{\left\langle n^{2}-m\right\rangle^{2(1-b)}}\right)^{1 / 2} \\
& =\sup _{\substack{v \in X^{0,1-b} \\
\|v\|_{0,1-b} \leq 1}}\left|\int_{0}^{1} \int_{B} v(t, x)(\sqrt{-\Delta})^{s} f(t, x) d x d t\right| \\
& \lesssim \sup _{\substack{v \in X^{0,1-b} \\
\|v\|_{0,1-b} \leq 1}}\|v\|_{L_{x}^{3-\epsilon} L_{t}^{2}}\left\|(\sqrt{-\Delta})^{s} u\right\|_{L_{x}^{\frac{3-\epsilon}{1-\epsilon}} L_{t}^{6}}\|u\|_{L_{x}^{6-2 \epsilon} L_{t}^{6}}^{2}
\end{aligned}
$$


Now, invoking Lemma 2.4 (i) in the form

$$
\|v\|_{L_{x}^{3-\epsilon} L_{t}^{2}} \lesssim\|v\|_{0,1-b},
$$

and using Lemma 3.1 to estimate the norms of $u$,

$$
\left\|\int_{0}^{t} e^{i(t-\tau) \Delta} f(\tau) d \tau\right\|_{s, b} \lesssim \lambda^{3}
$$

for each $\lambda>0$ and all $\omega \in \Omega$ outside a set of measure $O\left(\exp \left(-c \lambda^{c}\right)\right)$.

We therefore have (adjusting the value of the constant $c$ as well as the implicit constant)

$$
\mu_{F}^{(N)}\left(\left\{\phi:\left\|\int_{0}^{t} e^{i(t-\tau) \Delta} f d \tau\right\|_{s, b}>\lambda\right\}\right) \lesssim \exp \left(-c \lambda^{c}\right) .
$$

To conclude, collecting (3.8) and (3.9),

$$
\mu_{F}^{(N)}\left(\left\{\phi:\|u\|_{s, b}>\lambda\right\}\right) \lesssim \exp \left(-c \lambda^{c}\right)
$$

which gives the desired inequality.

\section{The NONLINEAR TERM}

The main issue is an estimate on the ||$|\cdot|||$-norm of trilinear expressions of the form

$$
\int_{0}^{t} e^{i(t-\tau) \Delta} P_{N}\left[P_{N_{1}} U^{1} \overline{P_{N_{2}} u^{(2)}} P_{N_{3}} u^{(3)}\right](\tau) d \tau
$$

with $t<T$, where $U^{1}$ belongs to $X_{\||||| \mid}$and $u^{(2)}, u^{(3)}: \mathbb{R} \times B \rightarrow \mathbb{C}$ are solutions to truncated equations (1.1) for possibly different truncations $N^{(2)} \geq N_{2}, N^{(3)} \geq N_{3}$ and initial data

$$
\left.u^{(i)}\right|_{t=0}=P_{N^{(i)}}(\phi), \quad i=2,3 .
$$

In order to establish a contractive estimate on (4.1), $T$ will have to be chosen sufficiently small; more specifically, we shall require

$$
T \sim \frac{1}{\log N_{*}} \text { with } N_{*}=\max \left(N_{1}, N_{2}, N_{3}\right) .
$$

As will be clear later on, this choice of $T$ is essential in our argument due to the presence of a certain logarithmic divergence.

Our analysis is based on $L_{x}^{p} L_{t}^{q}$ norms as well as the norms $\|\cdot\|_{s, b}$ and $\||\cdot|\|$. Various contributions are considered, requiring different arguments. While the norms $\|\cdot\|_{s, b}$ and $\|\cdot|\||$ allow in particular for Fourier restrictions of the form $\chi_{\left[\left(n^{2}-m\right) \lesssim K\right]}$, these operations are in general not allowed for $L_{x}^{p} L_{t}^{q}$ norms. For this reason, certain care is required in organizing the argument.

We denote by $N, N_{i}, i=1,2,3$ integers of the form $2^{j}$ and $n \sim N_{i}$ means $N_{i} \leq n<2 N_{i}$. Denote $u_{2}=P_{N_{2}} u^{(2)}$ and $u_{3}=P_{N_{3}} u^{(3)}$. 
We start by applying Lemma 2.2 and estimate |||(4.1)||| by

$$
\int_{0}^{1} \int_{B} \bar{v}\left(P_{N_{1}} U^{1}\right) \bar{u}_{2} u_{3} d x d t
$$

with

$$
\||v|\| \leq 1
$$

By Cauchy-Schwarz,

$$
\text { (4.3) } \leq\left[\iint|v|^{2}\left|u_{2}\right|^{2} d x d t\right]^{\frac{1}{2}}\left[\iint\left|P_{N_{1}} U^{1}\right|^{2}\left|u_{3}\right|^{2} d x d t\right]^{\frac{1}{2}} .
$$

In each factor on the right-hand side of (4.4), $u^{(2)}$ and $u^{(3)}$ are obtained from the same truncated equation. This is essential for our analysis.

We have therefore reduced the estimate of (4.3) to estimating

$$
\iint \overline{P_{N} v} P_{N_{1}} v_{1} \overline{P_{N_{2}} u} P_{N_{3}} u
$$

with $u$ obtained from some truncated equation (1.1) and

$$
\||v|\| \leq 1, \quad\left\|\left|v_{1}\right|\right\| \leq 1 .
$$

Write (4.5) as

$$
\sum_{\substack{n \leq N, n_{i} \leq N_{i} \\ m-m_{1}+m_{2}-m_{3}=0}} \overline{\hat{v}(n, m)} v_{1}\left(n_{1}, m_{1}\right) \overline{\hat{u}\left(n_{2}, m_{2}\right)} \hat{u}\left(n_{3}, m_{3}\right) c(n, \bar{n})
$$

with

$$
c(n, \bar{n})=c\left(n, n_{1}, n_{2}, n_{3}\right) .
$$

Subdividing $\left[0, N_{i}\right]$ into dyadic intervals $\left[N_{i}^{\prime}, 2 N_{i}^{\prime}\right]$, we estimate

$$
\text { (4.6) } \leq \sum_{N_{2}^{\prime}, N_{3}^{\prime}}\left|\sum_{\substack{n \leq N, n_{1} \leq N_{1}, n_{2} \sim N_{2}^{\prime}, n_{3} \sim N_{3}^{\prime} \\ m-m_{1}+m_{2}-m_{3}=0}} c(n, \bar{n}) A_{n, m, \bar{n}, \bar{m}}\right|
$$

with

$$
A_{n, m, \bar{n}, \bar{m}}=\overline{\hat{v}(n, m)} \hat{v}_{1}\left(n_{1}, m_{1}\right) \overline{\hat{u}\left(n_{2}, m_{2}\right)} \hat{u}\left(n_{3}, m_{3}\right) .
$$

Fix $N_{2}^{\prime}, N_{3}^{\prime}$ and assume $N_{2}^{\prime} \geq N_{3}^{\prime}$. Set

$$
K=\left(N_{2}^{\prime}\right)^{10^{-3}}
$$

and define

$$
c_{K}(n, \bar{n})= \begin{cases}c(n, \bar{n}), & \text { if }\left|n^{2}-n_{1}^{2}+n_{2}^{2}-n_{3}^{2}\right|<10 K \\ 0, & \text { otherwise. }\end{cases}
$$

We now estimate

$$
\left|\sum_{\substack{n \leq N, n_{1} \leq N_{1}, n_{2} \sim N_{2}^{\prime}, n_{3} \sim N_{3}^{\prime} \\ m-m_{1}+m_{2}-m_{3}=0}} c(n, \bar{n}) A_{n, m, \bar{n}, \bar{m}}\right| \leq
$$




$$
\begin{aligned}
& \sum_{N^{\prime}, N_{1}^{\prime}}\left|\sum_{\substack{n \sim N^{\prime}, n_{1} \sim N_{1}^{\prime}, n_{2} \sim N_{2}^{\prime}, n_{3} \sim N_{3}^{\prime} \\
m-m_{1}+m_{2}-m_{3}=0,\left|m-n^{2}\right| \geq K}} c(n, \bar{n}) A_{n, m, \bar{n}, \bar{m}}\right| \\
& +\sum_{N^{\prime}, N_{1}^{\prime}}\left|\sum_{\substack{n \sim N^{\prime}, n_{1} \sim N_{1}^{\prime}, n_{2} \sim N_{2}^{\prime}, n_{3} \sim N_{3}^{\prime} \\
m-m_{1}+m_{2}-m_{3}=0,\left|m-n^{2}\right|<K,\left|m_{1}-n_{1}^{2}\right| \geq K}} c(n, \bar{n}) A_{n, m, \bar{n}, \bar{m}}\right| \\
& +\left|\sum_{\substack{n \leq N, n_{1} \leq N_{1}, n_{2} \sim N_{2}^{\prime}, n_{3} \sim N_{3}^{\prime} \\
m-m_{1}+m_{2}-m_{3}=0,\left|m-n^{2}\right|<K,\left|m_{1}-n_{1}^{2}\right|<K}} c(n, \bar{n}) A_{n, m, \bar{n}, \bar{m}}\right| .
\end{aligned}
$$

Making a further decomposition according to which $\left|n^{2}-n_{1}^{2}+n_{2}^{2}-n_{3}^{2}\right|>10 K$ or $\left|n^{2}-n_{1}^{2}+n_{2}^{2}-n_{3}^{2}\right| \lesssim 10 K$ in (4.11), the contribution of (4.11) may be evaluated by bounding

$$
\begin{aligned}
& \sum_{N^{\prime}, N_{1}^{\prime}}\left|\sum_{\substack{n \sim N^{\prime}, n_{i} \sim N_{i}^{\prime},\left|n^{2}-n_{1}^{2}+n_{2}^{2}-n_{3}^{2}\right|>10 K \\
m-m_{1}+m_{2}-m_{3}=0,\left|m-n^{2}\right|<K,\left|m_{1}-n_{1}^{2}\right|<K}} c(n, \bar{n}) A_{n, m, \bar{n}, \bar{m}}\right| \\
& +\left|\sum_{\substack{n \leq N, n_{1} \leq N_{1}, n_{2} \sim N_{2}^{\prime}, n_{3} \sim N_{3}^{\prime} \\
m-m_{1}+m_{2}-m_{3}=0}} c_{K}(n, \bar{n}) A_{n, m, \bar{n}, \bar{m}}\right|
\end{aligned}
$$

where in (4.13) we replaced $\hat{v}$ by $\hat{v} \chi_{\left|m-n^{2}\right| \leq K}$ and $\hat{v}_{1}$ by $\hat{v}_{1} \chi_{\left|m_{1}-n_{1}^{2}\right|<K}$ (noting that the norm $\||\cdot|||$ is unconditional).

Note that if $n_{2}=n_{3}$ and $\left|n^{2}-n_{1}^{2}+n_{2}^{2}-n_{3}^{2}\right| \leq 10 K$, then either $n=n_{1}$ or $N^{\prime}+N_{1}^{\prime} \lesssim\left(N_{2}^{\prime}\right)^{10^{-3}}$. Hence, (4.13) is bounded by

$$
\begin{aligned}
& \sum_{N^{\prime}, N_{1}^{\prime}}\left|\sum_{\substack{n \sim N^{\prime}, n_{i} \sim N_{i}^{\prime}, n_{2} \neq n_{3} \\
m-m_{1}+m_{2}-m_{3}=0}} c_{K}(n, \bar{n}) A_{n, m, \bar{n}, \bar{m}}\right| \\
& +\sum_{N^{\prime}, N_{1}^{\prime} \lesssim\left(N_{2}^{\prime}\right)^{10-3}}\left|\sum_{\substack{n \sim N^{\prime}, n_{1} \sim N_{1}^{\prime}, n_{2} \sim N_{2}^{\prime} \\
m-m_{1}+m_{2}-m_{3}=0}} c_{K}\left(n, n_{1}, n_{2}, n_{2}\right) A_{n, m,\left(n_{1}, n_{2}, n_{2}\right), \bar{m}}\right| \\
& +\left|\sum_{\substack{n \leq N, n_{2} \sim N_{2}^{\prime} \\
m-m_{1}+m_{2}-m_{3}=0}} c\left(n, n, n_{2}, n_{2}\right) A_{n, m,\left(n, n_{2}, n_{2}\right), \bar{m}}\right|
\end{aligned}
$$

Let

$$
\sigma_{n, N_{2}^{\prime}}=\sum_{n_{2} \sim N_{2}^{\prime}} \frac{1}{n_{2}^{2}} c\left(n, n, n_{2}, n_{2}\right)=O(1)
$$

and estimate (4.16) by

$$
\sum_{N^{\prime}}\left|\sum_{n \sim N^{\prime}} \int_{0}^{1} \overline{\hat{v}(n)(\tau)} \hat{v}_{1}(n)(\tau)\left[\sum_{n_{2} \sim N_{2}^{\prime}} c\left(n, n, n_{2}, n_{2}\right)|\hat{u}(n)(\tau)|^{2}-\sigma_{n, N_{2}^{\prime}}\right] d \tau\right|
$$


ALMOST SURE GLOBAL WELL POSEDNESS FOR RADIAL NLS ON THE 3D BALL 21

$$
+\left|\sum_{n \leq N}\left(\int_{0}^{1} \overline{\hat{v}(n)(\tau)} \hat{v}_{1}(n)(\tau) d \tau\right) \sigma_{n, N_{2}^{\prime}}\right| .
$$

In view of the above observations, our estimate of |||(4.1) ||| reduces to establishing bounds on (4.9), (4.10), (4.12), (4.14), (4.15), (4.17) and (4.18); this will be the topic of the following two sections.

The choice of $T$ is dictated by (4.18), and we treat this term first. Indeed, taking $T$ sufficiently small, Lemma 2.3 gives

$$
\begin{aligned}
& \left|\sum_{n \leq N, m} \frac{\bar{v}(n, m)}{\hat{v}_{1}}(n, m) \sigma_{n, N_{2}^{\prime}}\right| \\
& \quad \lesssim \sum_{n \leq N, m}|\hat{v}(n, m)|\left|\hat{v}_{1}(n, m)\right| \lesssim T=o\left(\frac{1}{\log N_{*}}\right) .
\end{aligned}
$$

Evaluating the summation over dyadic $N_{2}^{\prime} \leq N_{*}$ then allows us to conclude that the contribution of (4.18) can be estimated by $o(1)$.

\section{Multilinear estimates (I)}

In this section, we obtain bounds on the terms (4.9), (4.10) and (4.12). The remaining terms will be treated in the next section.

We begin with the contribution of (4.9). Fix the values $N^{\prime}, N_{1}^{\prime}$ and rewrite the inner sum in (4.9) as

$$
\int_{B} \int_{0}^{1} \overline{\left(P_{n \sim N^{\prime}} v\right)}\left(P_{n_{1} \sim N_{1}^{\prime}} v_{1}\right) \overline{\left(P_{n_{2} \sim N_{2}^{\prime}} u\right)}\left(P_{n_{3} \sim N_{3}^{\prime}} u\right) d t d x
$$

where $\hat{v}(n, m)=0$ for $\left|m-n^{2}\right|<K$.

It follows from the definition of the ||$|\cdot|||$ norm that

$$
\left\|P_{n \sim N^{\prime}} v\right\|_{0, \frac{1}{3}}<K^{-\frac{1}{7}} \mid\left\|P_{n \sim N^{\prime}} v\right\| \| .
$$

Moreover, by (viii) from Lemma 2.4. applied with $b=\frac{1}{4}+\frac{3 \epsilon}{2}, \epsilon=10^{-6}$, we therefore obtain

$$
\left\|P_{n \sim N^{\prime}} v\right\|_{L_{x}^{1+\epsilon}} \frac{2}{L_{t}^{1-\epsilon}}<K^{-\frac{1}{7}}\left|\left\|P_{n \sim N^{\prime}} v \mid\right\| .\right.
$$

Also by (viii) of Lemma 2.4

$$
\left\|P_{n_{1} \sim N_{1}^{\prime}} v_{1}\right\|_{L_{x}^{1+\epsilon}}^{\frac{3}{1+\epsilon}} L_{t}^{\frac{2}{1-\epsilon}} \lesssim\left|\left\|P_{n \sim N_{1}^{\prime}} v_{1}\right\|\right|
$$

To estimate the contributions of $u_{2}$ and $u_{3}$ to (5.1), we use the apriori bound given by Lemma 3.1 with $q=\frac{2}{\epsilon}$, where $\epsilon=10^{-6}$ as before. In particular, we may ensure that

$$
\max \left\{\left\|P_{n_{2} \sim N_{2}^{\prime}} u\right\|_{L_{x}^{6-\epsilon} L_{t}^{q}},\left\|P_{n_{3} \sim N_{3}^{\prime}} u\right\|_{L_{x}^{6-\epsilon} L_{t}^{q}}\right\}<\left(N_{2}^{\prime}\right)^{10^{-6}}
$$


outside an exceptional set of measure at most $\exp \left(-c\left(N_{2}^{\prime}\right)^{c 10^{-6}}\right)$ in the initial datum $\phi$. Taking $p=\frac{6}{1-2 \epsilon}$, we then obtain

$$
\left\|P_{n_{2} \sim N_{2}^{\prime}} u\right\|_{L_{x}^{p} L_{t}^{q}} \lesssim\left(N_{2}^{\prime}\right)^{10^{-6}+\frac{3}{6-\epsilon}-\frac{1}{2}+\epsilon} .
$$

Hence, from (5.2)-(5.5) and recalling that $K=\left(N_{2}^{\prime}\right)^{10^{-3}}$ and $\epsilon=10^{-6}$, it follows that

$$
\begin{aligned}
\text { (5.1) } & <K^{-\frac{1}{7}}\left(N_{2}^{\prime}\right)^{10^{-6}+\frac{3}{6-\epsilon}-\frac{1}{2}+\epsilon}||\left|P_{n \sim N^{\prime}} v\right|\left\|\left|\| P_{n \sim N_{1}^{\prime}} v_{1}\right|||\right. \\
& <\left(N_{2}^{\prime}\right)^{-\frac{1}{2} 10^{-4}}||\left|P_{n \sim N^{\prime}} v\right|\left\|\left|\left\|P_{n \sim N_{1}^{\prime}} v_{1}\right\|\right| .\right.
\end{aligned}
$$

To complete the estimate of the contribution of (4.9), it remains to perform dyadic summation over $N^{\prime}, N_{1}^{\prime}, N_{2}^{\prime}$ and $N_{3}^{\prime}$, with $N_{2}^{\prime} \geq N_{3}^{\prime}$. Note that from the definition of the $\||\cdot|\|$ norm, one has

$$
\left.\||v|\|^{2} \sim \sum_{N^{\prime}}\left\|P_{n \sim N^{\prime}} v\right\|\right|^{2} .
$$

In view of (5.6), there is of course no problem with the summation over values of $N_{2}^{\prime}$ and $N_{3}^{\prime}$, and we may also assume $\max \left\{N^{\prime}, N_{1}^{\prime}\right\}>\exp \left(\left(N_{2}^{\prime}\right)^{10^{-5}}\right)$. Consider the case $N^{\prime} \geq N_{1}^{\prime}$. If $N^{\prime} \sim N_{1}^{\prime}$, the estimate follows by using Cauchy-Schwarz and (5.7) for $v$ and $v_{1}$. Assume now that $N^{\prime}>4 N_{1}^{\prime}$ holds. We estimate the contribution of such terms to (4.9) by

$$
\gamma \int\left[\sum_{n \sim N^{\prime}}|\hat{v}(n)(t)|\right]\left[\sum_{n_{1} \sim N_{1}^{\prime}}\left|\hat{v}_{1}\left(n_{1}\right)(t)\right|\right]\left[\sum_{n_{2} \sim N_{2}^{\prime}}\left|\hat{u}\left(n_{2}\right)(t)\right|\right]\left[\sum_{n_{3} \sim N_{3}^{\prime}}\left|\hat{u}\left(n_{3}\right)(t)\right|\right] d t
$$

with

$$
\gamma=\max _{n \sim N^{\prime}, n_{i} \sim N_{i}^{\prime}}\left|c\left(n, n_{1}, n_{2}, n_{3}\right)\right| .
$$

We then have the bound

$$
\begin{aligned}
\text { (5.8) } & \leq \gamma \cdot\left(N^{\prime} N_{1}^{\prime} N_{2}^{\prime} N_{3}^{\prime}\right)^{\frac{1}{2}}\|v\|_{L_{t}^{2} L_{x}^{2}}\left\|v_{1}\right\|_{L_{t}^{2} L_{x}^{2}}\|u\|_{L_{t}^{\infty} L_{x}^{2}}^{2} \\
& \leq \gamma \cdot\left(N^{\prime} N_{1}^{\prime} N_{2}^{\prime} N_{3}^{\prime}\right)^{\frac{1}{2}}\|\| v\|\|\left\|v_{1}\right\|\|\| u \|_{L_{t}^{\infty} L_{x}^{2} .}^{2}
\end{aligned}
$$

To evaluate $\gamma$, we write

$$
\int_{B} e_{n} e_{n_{1}} e_{n_{2}} e_{n_{3}} d x=\int_{0}^{1} \sin (n \pi r) \sin \left(n_{1} \pi r\right) \varphi(r) d r
$$

with $\varphi(r)=\frac{\sin \left(\pi n_{2} r\right)}{r} \cdot \frac{\sin \left(\pi n_{3} r\right)}{r}$, and note that integration by parts gives

$$
\begin{aligned}
\int_{0}^{1} \cos \left(\left(n \pm n_{1}\right) \pi r\right) \varphi(r) d r & =-\frac{1}{\pi\left(n \pm n_{1}\right)} \int_{0}^{1} \varphi^{\prime}(r) \sin \left(\left(n \pm n_{1}\right) \pi r\right) d r \\
& <O\left(\frac{\left\|\varphi^{\prime}\right\|_{L^{\infty}}}{\left(n \pm n_{1}\right)^{2}}\right) \\
& <O\left(\frac{\left(N_{2}^{\prime}\right)^{2} N_{3}^{\prime}}{\left(N^{\prime}\right)^{2}}\right)
\end{aligned}
$$


where the last line follows from $N^{\prime}>4 N_{1}^{\prime}$. Hence

$$
(5.9) \lesssim \frac{\left(N_{2}^{\prime}\right)^{4}}{N^{\prime}}
$$

Summing (5.9) over dyadic $N^{\prime}, N_{1}^{\prime}, N_{2}^{\prime}$ and $N_{3}^{\prime}$ satisfying $N^{\prime}>\max \left\{\exp \left(\left(N_{2}^{\prime}\right)^{10^{-5}}\right)\right.$, $\left.4 N_{1}^{\prime}\right\}$ and $N_{3}^{\prime} \leq N_{2}^{\prime}$, the contribution of (5.8) is then bounded by

$$
\sum_{N^{\prime}, N_{2}^{\prime}} \frac{\left(N_{2}^{\prime}\right)^{4}\left(\log N_{2}^{\prime}\right)\left(\log N^{\prime}\right)}{N^{\prime}}<\frac{1}{N_{2}^{\prime}},
$$

which completes the estimate of the contribution of (4.9).

Since $v$ and $v_{1}$ play the same role, the same argument also takes care of contribution of (4.10).

We now address the contribution of (4.12). Since the estimate relies only on $X_{s, b}$ norms, Fourier restrictions are not an issue. Note that since $\left|m-n^{2}\right|<K$, $\left|m_{1}-n_{1}^{2}\right|<K$ and $\left|n^{2}-n_{1}^{2}+n_{2}^{2}-n_{3}^{2}\right|>10 K$, at least one of the conditions

$$
\left|m_{2}-n_{2}^{2}\right|>K \quad \text { or } \quad\left|m_{3}-n_{3}^{2}\right|>K
$$

holds.

Assume

$$
\left|m_{2}-n_{2}^{2}\right| \gtrsim\left|m_{3}-n_{3}^{2}\right|>K
$$

We distinguish several cases.

Case 1: $N^{\prime}+N_{1}^{\prime}<\left(N_{2}^{\prime}\right)^{3}$.

Consider the expression

$$
\sum_{\substack{n \sim N^{\prime}, n_{i} \sim N_{i}^{\prime},\left|n^{2}-n_{1}^{2}+n_{2}^{2}-n_{3}^{2}\right| \gtrsim K \\ m-m_{1}+m_{2}-m_{3}=0 \\\left|m_{2}-n_{2}^{2}\right| \gtrsim\left|m_{3}-n_{3}^{2}\right|>K}} c(n, \bar{n}) A_{n, m, \bar{n}, \bar{m}}
$$

where we assume $\left|\left\|v\left|\left\|,\left|\left\|v_{1} \mid\right\| \leq 1\right.\right.\right.\right.\right.$ and, according to Proposition 3.2, that $\|u\|_{\frac{1}{2}-, \frac{3}{4}-}<$ $O(1)$.

The restriction $\left|n^{2}-n_{1}^{2}+n_{2}^{2}-n_{3}^{2}\right| \gtrsim K$ in (5.12) may be removed arguing as follows: Let $0 \leq \psi \leq 1$ be a parameter, and replace $\hat{v}(n, m)$ by

$$
e\left(n^{2} \psi\right) \hat{v}(n, m),
$$

and $\hat{v}_{1}\left(n_{1}, m_{1}\right), \hat{u}\left(n_{2}, m_{2}\right)$ and $\hat{u}\left(n_{3}, m_{3}\right)$ by

$$
e\left(n_{1}^{2} \psi\right) \hat{v}_{1}\left(n_{1}, m_{1}\right), \quad e\left(n_{2}^{2} \psi\right) \hat{u}\left(n_{2}, m_{2}\right) \quad \text { and } \quad e\left(n_{3}^{2} \psi\right) \hat{u}\left(n_{3}, m_{3}\right),
$$

respectively. The restriction $\left|n^{2}-n_{1}^{2}+n_{2}^{2}-n_{3}^{2}\right| \lesssim K$ may then be achieved by taking a suitable average over $\psi$. 
It thus suffices to bound the expression

$$
\sum_{\substack{n \sim N^{\prime}, n_{i} \sim N_{i}^{\prime}, m-m_{1}+m_{2}-m_{3}=0 \\\left|m_{2}-n_{2}^{2}\right| \gtrsim\left|m_{3}-n_{3}^{2}\right|>K}} c(n, \bar{n}) \overline{\hat{v}(n, m)} \hat{v}_{1}\left(n_{1}, m_{1}\right) \overline{\hat{u}_{2}\left(n_{2}, m_{2}\right)} \hat{u}_{3}\left(n_{3}, m_{3}\right)
$$

with $\left|\|v\|\left\|,||\left|v_{1}\right|\right\| \leq 1,\left\|u_{2}\right\|_{\frac{1}{2}-, \frac{3}{4}-}<O(1)\right.$ and $\left\|u_{3}\right\|_{\frac{1}{2}-, \frac{3}{4}-}<O(1)$.

To bound this quantity, we re-express (5.13) as

$$
\int_{B} \int_{0}^{1} \overline{K^{-\epsilon}\left(P_{n \sim N^{\prime}} v\right)}\left[K^{-\epsilon}\left(P_{n_{1} \sim N_{1}^{\prime}} v_{1}\right)\right] \overline{K^{2 \epsilon}\left(P_{n_{2} \sim N_{2}^{\prime}} u_{2}\right)}\left[P_{n_{3} \sim N_{3}^{\prime}} u_{3}\right] d t d x
$$

with $\epsilon=10^{-6}$, where to simplify notation we have suppressed an additional Fourier restriction on the $u_{2}$ and $u_{3}$ factors.

Since the norm $\||\cdot|\|$ indeed controls the norm $\|\cdot\|_{0, \frac{1}{2}-}$, and the condition $K^{\epsilon}>\left(N^{\prime}\right)^{\frac{1}{3} 10^{-9}}$ holds by assumption, we may apply inequality (iii) of Lemma 2.4 to obtain

and, similarly,

$$
\left\|K^{-\epsilon} P_{n \sim N^{\prime}} v\right\|_{L_{x}^{3} L_{t}^{4-}}<O(1)
$$

$$
\left\|K^{-\epsilon} P_{n_{1} \sim N_{1}^{\prime}} v_{1}\right\|_{L_{x}^{3} L_{t}^{4-}}<O(1) .
$$

On the other hand, using the Fourier restriction due to (5.11),

$$
\begin{aligned}
& \left\|P_{n_{2} \sim N_{2}^{\prime}} u_{2}\right\|_{\frac{1}{2}-, \frac{5}{8}}<K^{-\frac{1}{16}}\left\|u_{2}\right\|_{\frac{1}{2}-, \frac{3}{4}-}, \\
& \left\|P_{n_{2} \sim N_{2}^{\prime}} u_{2}\right\|_{\frac{1}{2}+\epsilon, \frac{5}{8}}<K^{-\frac{1}{16}}\left(N_{2}^{\prime}\right)^{\epsilon}\left\|u_{2}\right\|_{\frac{1}{2}-, \frac{3}{4}-} .
\end{aligned}
$$

Applying Lemma 2.4 (v), it follows that

$$
\left\|P_{n_{2} \sim N_{2}^{\prime}} u_{2}\right\|_{L_{x}^{p} L_{t}^{q}} \lesssim K^{-\frac{1}{16}}\left(N_{2}^{\prime}\right)^{\epsilon}\left\|u_{2}\right\|_{\frac{1}{2}-, \frac{3}{4}-}
$$

with

$$
p=\frac{6}{1-\frac{\epsilon}{2}}, \quad q=\frac{4}{1-\frac{\epsilon}{2}} .
$$

In addition, Lemma 2.4 (v) gives

$$
\left\|P_{n_{3} \sim N_{3}^{\prime}} u_{3}\right\|_{L_{x}^{6-} L_{t}^{4-}}<O(1) .
$$

Combining (5.15)-(5.19), we obtain

$$
|(\underline{5.14})| \lesssim\left(N_{2}^{\prime}\right)^{-\frac{10^{-3}}{16}+10^{-6}+2 \cdot 10^{-9}} \lesssim\left(N_{2}^{\prime}\right)^{-10^{-5}} .
$$

Summing in $N^{\prime}$ and $N_{1}^{\prime}$ now gives the bound

$$
\left(N_{2}^{\prime}\right)^{-10^{-5}}\left[\log \left(N_{2}^{\prime}\right)\right]^{2} \lesssim\left(N_{2}^{\prime}\right)^{-\frac{10^{-5}}{2}}
$$

for the contribution of these terms to (4.12).

Case 2: $N^{\prime}+N_{1}^{\prime}>\left(N_{2}^{\prime}\right)^{3}$ and $n \neq n_{1}$.

In this case, we have

$$
N^{\prime}+N_{1}^{\prime}-\left(N_{2}^{\prime}\right)^{2}<\left|n^{2}-n_{1}^{2}+n_{2}^{2}-n_{3}^{2}\right|<\left|n_{2}^{2}-m_{2}\right|+\left|n_{3}^{2}-m_{3}\right|+2 K
$$


and hence

$$
\left|n_{2}^{2}-m_{2}\right|>\frac{1}{3}\left(N^{\prime}+N_{1}^{\prime}\right)
$$

This clearly allows us to repeat the analysis of Case 1 with $\frac{1}{3}\left(N^{\prime}+N_{1}^{\prime}\right)$ in place of $K$, giving again the bound

$$
\left(N_{2}^{\prime}\right)^{-10^{-5}}
$$

Case 3: $N^{\prime}=N_{1}^{\prime}>\left(N_{2}^{\prime}\right)^{3}, n=n_{1}$.

Proceeding as in Case 1 above, we obtain

$$
\sum_{n \sim N^{\prime}, n_{i} \sim N_{i}^{\prime}, m-m_{1}+m_{2}-m_{3}=0} c\left(n, n, n_{2}, n_{3}\right) A_{n, m,\left(n, n_{2}, n_{3}\right), \bar{m}}
$$

with $\left|m_{2}-n_{2}^{2}\right|>K$. Rewrite (5.20) as

$$
\int_{B} \int_{0}^{1}\left[\sum_{n \sim N^{\prime}} \overline{\hat{v}(n)} \hat{v}_{1}(n) e_{n}^{2}\right] \overline{\left(P_{n_{2} \sim N_{2}^{\prime}} u_{2}\right)}\left(P_{n_{3} \sim N_{3}^{\prime}} u_{3}\right) d t d x .
$$

Now, observe that it follows from (5.17) and (5.18) that

$$
\left\|P_{n_{2} \sim N_{2}^{\prime}} u_{2}\right\|_{L_{x}^{p} L_{t}^{q}}<\left(N_{2}^{\prime}\right)^{-10^{-5}}
$$

while (5.19) gives

$$
\left\|P_{n_{3} \sim N_{3}^{\prime}} u_{3}\right\|_{L_{x}^{6-} L_{t}^{4-}}<O(1) .
$$

On the other hand, since $e_{n}^{2}(x) \leq \frac{1}{|x|^{2}}$, the first factor in the integrand of (5.21) is bounded by

$$
\frac{1}{|x|^{2}}\left(\sum_{n \sim N^{\prime}}|\hat{v}(n)|^{2}\right)^{\frac{1}{2}}\left(\sum_{n \sim N^{\prime}}\left|\hat{v}_{1}(n)\right|^{2}\right)^{\frac{1}{2}}
$$

where for any $q_{1}<\infty$ one has

$$
\left\|\left(\sum_{n}|\hat{v}(n)|^{2}\right)^{\frac{1}{2}}\right\|_{L_{t}^{q_{1}}}=\|v\|_{L_{t}^{q_{1}} L_{x}^{2}} \lesssim\|v\|_{0, \frac{1}{2}-} \lesssim\|\| v\|\|,
$$

with the analogous bound for $v_{1}$.

It then follows that

$$
\|\left(\underline{5.24)} \|_{L_{x}^{\frac{3}{2}-}-L_{t}^{q_{1}}}<O(1) .\right.
$$

Combining (5.25) with (5.22) along with (5.23) and summing in $N^{\prime}$ now gives that the contribution of (5.20) is bounded by

$$
\left(N_{2}^{\prime}\right)^{-10^{-5}},
$$

completing the bound in this case. 


\section{Multilinear estimates (II)}

In this section, we estimate the remaining contributions, those of (4.14), (4.15) and (4.17). This will involve a different type of analysis than that used in the previous section; in particular we will make essential use of several further probabilistic considerations related to the solution map.

We begin with (4.14). Rewrite this quantity as a sum over $N^{\prime}, N_{1}^{\prime}$ of

$$
\left|\int_{0}^{1}\left[\sum_{n \sim N^{\prime}, n_{i} \sim N_{i}^{\prime}, n_{2} \neq n_{3}} c_{K}(n, \bar{n}) \overline{\hat{v}(n)} \hat{v}_{1}\left(n_{1}\right) \overline{\hat{u}\left(n_{2}\right)} \hat{u}\left(n_{3}\right)\right] d t\right| .
$$

Note that in the sum we necessarily have $n \neq n_{1}$, since otherwise

$$
N_{2}^{\prime}+N_{3}^{\prime} \leq\left|n_{2}^{2}-n_{3}^{2}\right| \leq 10 K=10\left(N_{2}^{\prime}\right)^{10^{-3}},
$$

giving a contradiction.

Hence, it follows that

$$
N^{\prime}+N_{1}^{\prime} \leq\left|n^{2}-n_{1}^{2}\right| \leq K+8\left(N_{2}^{\prime}\right)^{2}<9\left(N_{2}^{\prime}\right)^{2} .
$$

We first examine the contribution for $n \neq n_{3}$. Denote $N^{\prime}, N_{i}^{\prime}$ by $N, N_{i}$ for simplicity. Since $\|v\|_{L_{t, x}^{2}} \lesssim 1$, it follows from Cauchy-Schwarz that (6.1) is bounded by the $L_{t}^{2}$-norm of

$$
\begin{aligned}
{\left[\sum_{n} \mid \sum_{n_{1}, n_{2}, n_{3}} \hat{v}_{1}\left(n_{1}\right)\right.} & \left.\left.\overline{\hat{u}\left(n_{2}\right)} \hat{u}\left(n_{3}\right) c_{K}\left(n, n_{1}, n_{2}, n_{3}\right)\right|^{2}\right]^{\frac{1}{2}} \\
\leq & \leq\left[\sum_{n_{1}, n_{1}^{\prime}}\left|\hat{v}_{1}\left(n_{1}\right)\right|\left|\hat{v}_{1}\left(n_{1}^{\prime}\right)\right| \sum_{\substack{n, n_{2}, n_{2}^{\prime}, n_{3}, n_{3}^{\prime}}} B_{n, \bar{n}_{,}, \bar{n}^{\prime}} \mid\right]^{\frac{1}{2}}
\end{aligned}
$$

where

$$
B_{n, \bar{n}, \bar{n}^{\prime}}=\overline{\hat{u}\left(n_{2}\right)} \hat{u}\left(n_{3}\right) \hat{u}\left(n_{2}^{\prime}\right) \overline{\hat{u}\left(n_{3}^{\prime}\right)} c_{K}\left(n, n_{1}, n_{2}, n_{3}\right) c_{K}\left(n, n_{1}^{\prime}, n_{2}^{\prime}, n_{3}^{\prime}\right)
$$

and again by Cauchy-Schwarz

$$
\begin{array}{r}
{\left[\sum_{n_{1}}\left|\hat{v}_{1}\left(n_{1}\right)\right|^{2}\right]^{\frac{1}{2}}\left[\sum_{n_{1} \neq n_{1}^{\prime}}\left|B_{n, \bar{n}, \bar{n}^{\prime}}\right|^{2}\right]^{\frac{1}{4}}+\left[\sum_{n_{1}}\left|\hat{v}_{1}\left(n_{1}\right)\right|^{2}\right]^{\frac{1}{2}}\left[\max _{n_{1}=n_{1}^{\prime}}\left|B_{n, \bar{n}, \bar{n}^{\prime}}\right|\right]^{\frac{1}{2}}} \\
\leq\left\|v_{1}(t)\right\|_{L_{x}^{2}}\left\{\left[\sum_{n_{1} \neq n_{1}^{\prime}}\left|B_{n, \bar{n}, \bar{n}^{\prime}}\right|^{2}\right]^{\frac{1}{4}}+\max _{n_{1}=n_{1}^{\prime}}\left|B_{n, \bar{n}, \bar{n}^{\prime}}\right|^{\frac{1}{2}}\right\} .
\end{array}
$$

Since $\left\|v_{1}\right\|_{L_{t}^{q} L_{x}^{2}} \lesssim\left\||| v_{1} \mid\right\|<O(1)$ for all $q<\infty$, it suffices to bound

$$
\|\{\cdots\}\|_{L_{t}^{4}}
$$

where $\{\cdots\}$ is the quantity appearing in (6.2). 
Note that (6.3) involves only the truncated solution $u$ with initial data $\phi=\phi_{\omega}$, and we view $u$ as a random variable of $\omega$. For fixed $t$, the distribution of $u_{\omega}(t)$ is given by a Gaussian Fourier series

$$
\sum_{n} \frac{g_{n}(\omega)}{n} e_{n}
$$

with $\left\{g_{n}\right\}$ as a sequence of IID normalized complex Gaussians. This fact is essential to our analysis in this section.

For sufficiently large $q$, we may estimate

$$
\left(\mathbb{E}_{\omega}\left[\|\{\cdots\}\|_{L_{t}^{4}}^{q}\right]\right)^{\frac{1}{q}} \leq\|\|\{\cdots\}\left\|_{L_{\omega}^{q}}\right\|_{L_{t}^{4}} \leq \max _{0 \leq t \leq 1}\|\{\cdots\}\|_{L_{\omega}^{q}}
$$

and, fixing $t$, we accordingly write

$$
\begin{aligned}
& \|\{\cdots\}\|_{L_{\omega}^{q} \leq} \\
& \quad\left\{\sum_{n_{1} \neq n_{1}^{\prime}}\left\|\sum_{n, n_{2}, n_{3}, n_{2}^{\prime}, n_{3}^{\prime}} \frac{\overline{g_{n_{2}}}}{n_{2}} \frac{g_{n_{3}}}{n_{3}} \frac{g_{n_{2}^{\prime}}}{n_{2}^{\prime}} \frac{\overline{g_{n_{3}^{\prime}}}}{n_{3}^{\prime}} c_{K}(n, \bar{n}) c_{K}\left(n, \bar{n}^{\prime}\right)\right\|_{L_{\omega}^{q / 2}}^{2}\right\}^{\frac{1}{4}} \\
& \quad+\left\|\max _{n_{1}} \mid \sum_{n, n_{2}, n_{3}, n_{2}^{\prime}, n_{3}^{\prime}} \frac{\overline{g_{n_{2}}}}{n_{2}} \frac{g_{n_{3}}}{n_{3}} \frac{g_{n_{2}^{\prime}}}{n_{2}^{\prime}} \frac{\overline{g_{n_{3}^{\prime}}}}{n_{3}^{\prime}} c_{K}(n, \bar{n}) c_{K}\left(n, n_{1}, n_{2}^{\prime}, n_{3}^{\prime}\right)\right\|_{L_{\omega}^{q / 2}}^{\frac{1}{2}}
\end{aligned}
$$

We first analyze (6.4) by considering several cases, recalling that $n_{2} \neq n_{3}$ and $n_{2}^{\prime} \neq n_{3}^{\prime}$.

Case 1: $n_{2} \neq n_{2}^{\prime}, n_{3} \neq n_{3}^{\prime}$.

In this case, we note that the bound

$$
c_{K}\left(n, n_{1}, n_{2}, n_{3}\right) \lesssim N_{3} \chi_{\left[\left|n^{2}-n_{1}^{2}+n_{2}^{2}-n_{3}^{2}\right|<K\right]}
$$

gives the estimate

$$
\begin{aligned}
\mathbb{E}_{\omega}\left[\left|\sum_{n, n_{2}, n_{3}, n_{2}^{\prime}, n_{3}^{\prime}} \frac{\overline{g_{n_{2}}}}{n_{2}} \frac{g_{n_{3}}}{n_{3}} \frac{g_{n_{2}^{\prime}}}{n_{2}^{\prime}} \frac{\overline{g_{n_{3}^{\prime}}}}{n_{3}^{\prime}} c_{K}(n, \bar{n}) c_{K}\left(n, \bar{n}^{\prime}\right)\right|^{2}\right] \\
\quad \lesssim \frac{1}{N_{2}^{4}} \sum_{n_{2}, n_{2}^{\prime}, n_{3}, n_{3}^{\prime}}\left(\sum_{n} \chi_{\left|n^{2}-n_{1}^{2}+n_{2}^{2}-n_{3}^{2}\right|<K} \chi_{\left.\left|n^{2}-\left(n_{1}^{\prime}\right)^{2}+\left(n_{2}^{\prime}\right)^{2}-\left(n_{3}^{\prime}\right)^{2}\right|<K\right)^{2}}\right. \\
\quad \frac{\sqrt{K}}{N_{2}^{4}} \sum_{n, n_{2}, n_{2}^{\prime}, n_{3}, n_{3}^{\prime}} \chi_{\left|n^{2}-n_{1}^{2}+n_{2}^{2}-n_{3}^{2}\right|<K} \chi_{\left|n^{2}-\left(n_{1}^{\prime}\right)^{2}+\left(n_{2}^{\prime}\right)^{2}-\left(n_{3}^{\prime}\right)^{2}\right|<K .}
\end{aligned}
$$

For the summation over $n_{1}$ and $n_{1}^{\prime}$ in (6.4), this gives the bound

$$
\begin{aligned}
& \frac{\sqrt{K}}{N_{2}^{4}} \mid\left\{\left(n, n_{1}, n_{1}^{\prime}, n_{2}, n_{2}^{\prime}, n_{3}, n_{3}^{\prime}\right): n_{i}, n_{i}^{\prime} \sim N_{i}, n \neq n_{1}, n_{1}^{\prime},\right. \text { and } \\
& \left.\quad\left|n^{2}-n_{1}^{2}+n_{2}^{2}-n_{3}^{2}\right|<K,\left|n^{2}-\left(n_{1}^{\prime}\right)^{2}+\left(n_{2}^{\prime}\right)^{2}-\left(n_{3}^{\prime}\right)^{2}\right|<K\right\} \mid
\end{aligned}
$$


Fix values of $k, k^{\prime}$ with $|k|,\left|k^{\prime}\right|<K$, and evaluate the number of solutions of the equations

$$
\left\{\begin{array}{l}
n^{2}-n_{1}^{2}+n_{2}^{2}-n_{3}^{2}=k \\
n^{2}-\left(n_{1}^{\prime}\right)^{2}+\left(n_{2}^{\prime}\right)^{2}-\left(n_{3}^{\prime}\right)^{2}=k^{\prime}
\end{array}\right.
$$

in the variables $n, n_{1}, n_{1}^{\prime}, n_{2}, n_{2}^{\prime}, n_{3}$ and $n_{3}^{\prime}$.

For this purpose, further fix $n_{2}, n_{2}^{\prime}, n_{3}$. Since $n \pm n_{1}$ are divisors of $k-n_{2}^{2}+n_{3}^{2} \neq 0$, this specifies $n, n_{1}$ up to $N_{2}^{0+}$ possibilities. Next, writing

$$
\left(n_{1}^{\prime}\right)^{2}+\left(n_{3}^{\prime}\right)^{2}=n^{2}+\left(n_{2}^{\prime}\right)^{2}-k^{\prime}
$$

the usual bounds for the number of $\mathbb{Z}^{2}$-points on circles (and circle arcs) imply that (6.8) has at most $N_{3}^{0+}$ solutions in $\left(n_{1}^{\prime}, n_{3}^{\prime}\right)$.

Summarizing, this proves that

$$
\text { (6.6) }<\sqrt{K} N_{2}^{-4} K^{2} N_{2}^{2+} N_{3}<N_{2}^{-1 / 2}
$$

Case 2: $n_{2}=n_{2}^{\prime}, n_{3} \neq n_{3}^{\prime}$.

We obtain

$$
\begin{aligned}
\mathbb{E}_{\omega}\left[\left|\sum_{n, n_{2}, n_{3}, n_{3}^{\prime}} \frac{\left|g_{n_{2}}\right|^{2}}{\left(n_{2}\right)^{2}} \frac{g_{n_{3}}}{n_{3}} \frac{\overline{g_{n_{3}^{\prime}}}}{n_{3}^{\prime}} c_{K}(n, \bar{n}) c_{K}\left(n, n_{1}^{\prime}, n_{2}, n_{3}^{\prime}\right)\right|^{2}\right] \\
\quad \lesssim \frac{1}{N_{2}^{4}} \sum_{n_{3}, n_{3}^{\prime}}\left(\sum_{n, n_{2}} \chi_{\left|n^{2}-n_{1}^{2}+n_{2}^{2}-n_{3}^{2}\right|<K} \cdot \chi_{\left|n^{2}-\left(n_{1}^{\prime}\right)^{2}+n_{2}^{2}-\left(n_{3}^{\prime}\right)^{2}\right|<K}\right)^{2}
\end{aligned}
$$

and since the number of $\left(n, n_{2}\right)$-terms in the inner sum is at most $K N_{2}^{0+}$ (for given $\left.n_{1}, n_{1}^{\prime}, n_{3}, n_{3}^{\prime}\right)$, we obtain

$$
\text { (6.10) } \ll N_{2}^{-4+} K \sum_{n, n_{2}, n_{3}, n_{3}^{\prime}} \chi_{\left|n^{2}-n_{1}^{2}+n_{2}^{2}-n_{3}^{2}\right|<K} \chi_{\left|n^{2}-\left(n_{1}^{\prime}\right)^{2}+n_{2}^{2}-\left(n_{3}^{\prime}\right)^{2}\right|<K} \text {. }
$$

Taking the summation of (6.11) over $n_{1}$ and $n_{1}^{\prime}$ then gives the bound

$$
N_{2}^{-4+} K^{3} N_{2} N_{3}<N_{2}^{-1}
$$

for the contribution to the sum in (6.4).

Case 3: $n_{2} \neq n_{2}^{\prime}, n_{3}=n_{3}^{\prime}$.

In place of (6.10), we get

$$
\frac{1}{N_{2}^{4}} \sum_{n_{2}, n_{2}^{\prime}}\left(\sum_{n, n_{3}} \chi_{\left|n^{2}-n_{1}^{2}+n_{2}^{2}-n_{3}^{2}\right|<K} \cdot \chi_{\left|n^{2}-\left(n_{1}^{\prime}\right)^{2}+\left(n_{2}^{\prime}\right)-n_{3}^{2}\right|<K}\right)^{2} .
$$

Writing $n^{2}-n_{1}^{2}+n_{2}^{2}-n_{3}^{2}=k,|k|<K$, in the inner sum, it follows that $n \pm n_{3}$ divides $k+n_{1}^{2}-n_{2}^{2} \neq 0$, since $n \neq n_{3}$. Thus, there are at most $K N_{2}^{0+}$ terms in the inner sum and we obtain the bound

$$
N_{2}^{-4+} K^{3} N_{2}^{2} N_{3}<N_{2}^{-\frac{1}{2}}
$$

for the contribution of (6.12) to the sum in (6.4). 
Case 4: $n_{2}=n_{2}^{\prime}, n_{3}=n_{3}^{\prime}$.

In this case, the inner sum in (6.4) becomes

$$
N_{2}^{2} N_{3}^{-2} \sum_{n, n_{2}, n_{3}} c_{K}\left(n, n_{1}, n_{2}, n_{3}\right) c_{K}\left(n, n_{1}^{\prime}, n_{2}, n_{3}\right)
$$

It follows from the definition of $c_{K}$ that the quantity (6.13) vanishes unless

$$
\left|n_{1}^{2}-\left(n_{1}^{\prime}\right)^{2}\right|<2 K
$$

holds; note that this implies $N_{1}=O(K)$, since $n_{1} \neq n_{1}^{\prime}$. Thus

$$
\begin{aligned}
\text { (6.13) } & <N_{2}^{-2} \mid\left\{\left(n, n_{2}, n_{3}\right): n_{2} \sim N_{2}, n_{3} \sim N_{3} \text { and }\left|n^{2}+n_{2}^{2}-n_{3}^{2}\right| \lesssim K^{2}\right\} \mid \\
& \lesssim N_{2}^{-2} K^{2} N_{3} N_{2}^{0+} \\
& <N_{2}^{-\frac{3}{4}}
\end{aligned}
$$

and the corresponding contribution to (6.4) is bounded by $N_{2}^{-\frac{1}{4}}$.

The considerations in Cases 1-4 take care of the estimate of (6.4).

We next consider the estimate of (6.5). Note that the analogues of Cases 1, 2 and 3 in this setting are captured by the previous analysis, since we did not use the condition $n_{1} \neq n_{1}^{\prime}$.

To treat the estimate in the analogue of Case 4, we bound the contribution to (6.5) by

$$
\begin{aligned}
\left(\log N_{1}\right)\left[\max _{n_{1} \sim N_{1}}\right. & \left.N_{2}^{-2} N_{3}^{-2} \sum_{n, n_{2}, n_{3}} c_{K}\left(n, n_{1}, n_{2}, n_{3}\right)^{2}\right]^{\frac{1}{2}} \\
& \lesssim\left(\log N_{1}\right) N_{2}^{-1}\left[\max _{n_{1} \sim N_{1}} \sum_{n, n_{2}, n_{3}} \chi_{\left|n^{2}-n_{1}^{2}+n_{2}^{2}-n_{3}^{2}\right|<K}\right]^{\frac{1}{2}} \\
& \lesssim\left(\log N_{1}\right) N_{2}^{-1}\left(K N_{3} N_{2}^{0+}\right)^{\frac{1}{2}} \\
& \lesssim N_{2}^{-\frac{1}{3}}
\end{aligned}
$$

This completes the treatement of Case 4 for the estimate of (6.5). Combining the estimates of (6.4) and (6.5) then completes the analysis of the contribution of terms where $n \neq n_{3}$.

We now consider the terms for which $n_{3}=n$. Note that, since under this condition we have

$$
\left|n_{1}^{2}-n_{2}^{2}\right| \lesssim K=\left(N_{2}\right)^{10^{-3}}
$$

it also follows that $n_{1}=n_{2}$ in this setting. We then estimate the contribution to (6.1) by

$$
\min \left(N, N_{1}\right) \int\left[\sum_{n \sim N, n_{1} \sim N_{1}}|\hat{v}(n)|\left|\hat{v}_{1}\left(n_{1}\right)\right||\hat{u}(n)|\left|\hat{u}\left(n_{1}\right)\right|\right] d t
$$


which, after using Cauchy-Schwarz, is in turn estimated by

$$
\min \left(N, N_{1}\right) \int\left\|P_{n \sim N} v\right\|_{L_{x}^{2}}\left\|P_{n \sim N} u\right\|_{L_{x}^{2}}\left\|P_{n_{1} \sim N_{1}} v_{1}\right\|_{L_{x}^{2}}\left\|P_{n_{1} \sim N_{1}} u\right\|_{L_{x}^{2}} d t .
$$

Using Hölder and summing over $N$ and $N_{1}$, we obtain the bound

$$
\begin{aligned}
\sum_{N, N_{1}} \min \left\{N, N_{1}\right\}\left\|P_{n \sim N} v\right\|_{L_{t, x}^{2}}\left\|P_{n \sim N} u\right\|_{L_{t}^{6} L_{x}^{2}} \\
\cdot\left\|P_{n_{1} \sim N_{1}} v_{1}\right\|_{L_{t}^{6} L_{x}^{2}}\left\|P_{n_{1} \sim N_{1}} u\right\|_{L_{t}^{6} L_{x}^{2}} .
\end{aligned}
$$

Moreover, since \|\|$_{L_{t}^{q} L_{x}^{2}} \lesssim\|\| \cdot|| \mid$ holds for all $q$, and, by Lemma 2.3 .

$$
\left(\sum_{N}\left\|P_{n \sim N} v\right\|_{L_{x, t}^{2}}^{2}\right)^{\frac{1}{2}}=\|v\|_{L_{x, t}^{2}} \lesssim \sqrt{T}\|\mid v\| \|,
$$

it follows from Cauchy-Schwarz that

$$
\begin{aligned}
(\underline{6.14}) \lesssim \sqrt{T}\left\{\sum _ { N } \| P _ { n \sim N } u \| _ { L _ { t } ^ { 6 } L _ { x } ^ { 2 } } ^ { 2 } \left(\sum_{N_{1}} \min \left\{N, N_{1}\right\}\right.\right. \\
\left.\left.\cdot\left\|P_{n_{1} \sim N_{1}} v_{1}\right\|\left\|P_{n_{1} \sim N_{1}} u\right\|_{L_{t}^{6} L_{x}^{2}}\right)^{2}\right\}^{\frac{1}{2}} .
\end{aligned}
$$

To control the norms of projections of $u$ appearing in (6.15) we require the following probabilistic estimate.

Lemma 6.1. Let $1 \leq q<+\infty$ be given. Then there exists $c>0$ such that for every $\lambda \geq 1$, one has

$$
\mu_{F}^{(N)}\left(\left\{\phi: \max _{N} N^{1 / 2}\left\|P_{n \sim N} u_{\phi}\right\|_{L_{t}^{q} L_{x}^{2}}>\lambda\right\}\right) \leq \exp \left(-c \lambda^{c}\right)
$$

where the maximum is taken over dyadic integers $N$.

Assuming that Lemma 6.1 holds, we use this bound to estimate (6.15) by

$$
\sqrt{T}\left\{\sum_{N}\left(\sum_{N_{1}} \frac{\min \left\{N, N_{1}\right\}}{\sqrt{N N_{1}}}\left\|\mid P_{n_{1} \sim N_{1}} v_{1}\right\|\right)^{2}\right\}^{1 / 2} \lesssim \sqrt{T} \mid\left\|v_{1}\right\| .
$$

This leads to the bound $O(\sqrt{T})$ on (6.14).

This completes the analysis of the contribution of (4.14) except for the proof of Lemma 6.1, which we address presently.

Proof of Lemma 6.1. We begin by noting that it suffices to establish

$$
\mu_{G}\left(A_{\lambda}\right) \leq \exp \left(-c \lambda^{c}\right)
$$

with $A_{\lambda}:=\left\{\phi: \max _{N} N^{1 / 2}\left\|P_{n \sim N} u_{\phi}\right\|_{L_{t}^{q}\left(\left[0, T_{*}\right) ; L_{x}^{2}(B)\right)}>\lambda\right\}$. Indeed, arguing as in the proof of Lemma 3.1, (6.17) implies then an inequality of the type (6.16). 
It therefore remains to establish (6.17). Toward this end, fixing $q_{1}>q$ and applying the Tchebychev inequality and Plancherel identity followed by the Minkowski inequality, one obtains

$$
\begin{aligned}
\mu_{G}\left(A_{\lambda}\right) & \leq \lambda^{-q_{1}}\left\|\max _{N}\left(N^{1 / 2}\left\|P_{n \sim N} u_{\phi}\right\|_{L_{t}^{q} L_{x}^{2}}\right)\right\|_{L^{q_{1}}\left(d \mu_{G}\right)}^{q_{1}} \\
& \lesssim \lambda^{-q_{1}}\left\|\max _{N} N^{1 / 2}\left(\sum_{n \sim N}\left|\widehat{u}_{\phi}(n)\right|^{2}\right)^{1 / 2}\right\|_{L^{q_{1}}\left(d \mu_{G}\right)} \|_{L_{t}^{q}}^{q_{1}} \\
& \lesssim \lambda^{-q_{1}}\left\|\max _{N} N^{1 / 2}\left(\sum_{n \sim N}|\widehat{\phi}(n)|^{2}\right)^{1 / 2}\right\|_{L^{q_{1}}\left(d \mu_{G}\right)}^{q_{1}} \\
& \lesssim \lambda^{-q_{1}}\left\|\max _{N} N^{1 / 2}\left(\sum_{n \sim N} \frac{\left|g_{n}(\omega)\right|^{2}}{n^{2}}\right)^{1 / 2}\right\|_{L_{\omega}^{q_{1}}}^{q_{1}}
\end{aligned}
$$

where we used the invariance of the Gibbs measure to obtain the third inequality.

We therefore have

$$
\begin{aligned}
(16.18) & \lesssim \lambda^{-q_{1}}\left\{1+\left(\sum_{N}\left\|\sum_{n \sim N} \frac{N}{n^{2}}\left(\left|g_{n}(\omega)\right|^{2}-1\right)\right\|_{L_{\omega}^{q_{1}}}\right)^{1 / 2}\right\}^{q_{1}} \\
& \lesssim \lambda^{-q_{1}}\left\{1+\left(\sum_{N} \frac{q_{1}}{\sqrt{N}}\right)^{1 / 2}\right\}^{q_{1}} \\
& \lesssim\left(\frac{\sqrt{q_{1}}}{\lambda}\right)^{q_{1}}
\end{aligned}
$$

where we used the estimate

$$
\left\|\sum_{n \sim N} \frac{N}{n^{2}}\left(\left|g_{n}(\omega)\right|^{2}-1\right)\right\|_{L_{\omega}^{q_{1}}} \lesssim N q_{1}\left(\sum_{n \sim N} \frac{1}{n^{4}}\right)^{1 / 2} \lesssim \frac{q_{1}}{\sqrt{N}}
$$

which follows from (2.7). Optimizing the choice of $q_{1}$ (by essentially taking $q_{1}=$ $\lambda^{2} / 2$; see, for instance, the proof of Lemma 3.1), now yields the desired claim.

This completes the proof of Lemma 6.1.

It remains to bound the contributions of (4.15) and (4.17). We begin with (4.15), for which we argue by expressing the inner sum in this expression as

$$
\sum_{n \sim N, n_{1} \sim N_{1}, n_{2} \sim N_{2}} c_{K}\left(n, n_{1}, n_{2}, n_{2}\right) \int_{0}^{1}\left[\overline{\hat{v}(n)} \hat{v}_{1}\left(n_{1}\right)\left|\hat{u}\left(n_{2}\right)\right|^{2}\right] d t .
$$

Using Lemma 6.1, this is in turn bounded by

$$
\begin{aligned}
N \int_{0}^{1} & \left(\sum_{n \sim N}|\hat{v}(n)|\right)\left(\sum_{n_{1} \sim N_{1}}\left|\hat{v}_{1}\left(n_{1}\right)\right|\right)\left(\sum_{n_{2} \sim N_{2}}\left|\hat{u}\left(n_{2}\right)\right|^{2}\right) d t \\
& \leq N^{3 / 2} N_{1}^{1 / 2} \int_{0}^{1}\left\|P_{n \sim N} v\right\|_{L_{x}^{2}}\left\|P_{n_{1} \sim N_{1}} v_{1}\right\|_{L_{x}^{2}}\left\|P_{n_{2} \sim N_{2}} u\right\|_{L_{x}^{2}}^{2} d t \\
& \lesssim\left(N_{2}\right)^{2 \cdot 10^{-3}}\left\|P_{n \sim N} v\right\|_{L_{t}^{4} L_{x}^{2}}\left\|P_{n_{1} \sim N_{1}} v_{1}\right\|_{L_{t}^{4} L_{x}^{2}}\left\|P_{n_{2} \sim N_{2}} u\right\|_{L_{t}^{4} L_{x}^{2}}^{2}
\end{aligned}
$$




$$
\lesssim\left(N_{2}\right)^{2 \cdot 10^{-3}-1}
$$

We next consider (4.17). We use the Cauchy-Schwarz inequality to bound this expression by

$$
\begin{gathered}
\sum_{N}\left\|P_{n \sim N} v\right\|_{L_{t, x}^{2}}\left\|P_{n \sim N} v_{1}\right\|_{L_{t}^{4} L_{x}^{2}}\left\|\left.\max _{n \sim N}\left|\sum_{n_{2} \sim N_{2}} c\left(n, n, n_{2}, n_{2}\right)\right| \hat{u}\left(n_{2}\right)\right|^{2}-\sigma_{n, N_{2}} \mid\right\|_{L_{t}^{4}} \\
\lesssim T^{1 / 2} \sup _{N}\left\|\left.\max _{n \sim N}\left|\sum_{n_{2} \sim N_{2}} c\left(n, n, n_{2}, n_{2}\right)\right| \hat{u}\left(n_{2}\right)\right|^{2}-\sigma_{n, N_{2}} \mid\right\|_{L_{t}^{4}}
\end{gathered}
$$

Recall that

$$
\sigma_{n}=\sigma_{n, N_{2}}=\mathbb{E}_{\phi}\left[\sum_{n_{2} \sim N_{2}} c\left(n, n, n_{2}, n_{2}\right)\left|\hat{\phi}\left(n_{2}\right)\right|^{2}\right] .
$$

The bound on the second factor in (6.19) again follows from probabilistic considerations. We have the following:

Lemma 6.2. For $\lambda \gg 1$, we have for some constant $c>0$

$$
\mu_{F}\left[\phi ; \|\left.\max _{n}\left|\sum_{n_{2} \sim N_{2}}\right| \widehat{u_{\phi}(t)}\left(n_{2}\right)\right|^{2}\left(c\left(n, n, n_{2}, n_{2}\right)-\sigma_{n} \mid \|_{L_{t}^{4}}>\lambda\right] \lesssim e^{-c \lambda^{c} N_{2}^{c}}\right.
$$

Proof. It suffices again to prove (6.20) with $\mu_{F}$ replaced by the Gibbs measure $\mu_{G}$. Proceeding as in Lemma 6.1, take $q_{1}=q_{1}(\lambda)$ and write

$$
\begin{aligned}
\|\| \max _{n} \mid & \sum_{n_{2} \sim N_{2}}\left|\widehat{u_{\phi}(t)}\left(n_{2}\right)\right|^{2}\left(c\left(n, n, n_{2}, n_{2}\right)-\sigma_{n} \mid\left\|_{L_{t}^{4}}\right\|_{L^{q_{1}}\left(\mu_{G}(d \phi)\right)}\right. \\
& \leq\left.\|\| \max _{n}\left|\sum_{n_{2} \sim N_{2}}\right| \widehat{u_{\phi}(t)}\left(n_{2}\right)\right|^{2}\left(c\left(n, n, n_{2}, n_{2}\right)-\sigma_{n} \mid\left\|_{\left.L^{q_{1}\left(\mu_{G}(d \phi)\right)}\right)}\right\|_{L_{t}^{4}} .\right.
\end{aligned}
$$

Using the Gibbs measure invariance under the flow, the above is bounded by

$$
\begin{aligned}
& \left\|\left.\max _{n}\left|\sum_{n_{2} \sim N_{2}}\right| \hat{\phi}\left(n_{2}\right)\right|^{2} c\left(n, n, n_{2}, n_{2}\right)-\sigma_{n} \mid\right\|_{L^{q_{1}}\left(\mu_{G}(d \phi)\right)} \\
& \quad \leq\left\|\max _{n}\left|\sum_{n_{2} \sim N_{2}} \frac{c\left(n, n, n_{2}, n_{2}\right)}{n_{2}^{2}}\left(\left|g_{n_{2}}(\omega)\right|^{2}-1\right)\right|\right\|_{L^{q_{1}}(d \omega)} .
\end{aligned}
$$

Note that

$$
\begin{aligned}
c\left(n, n, n_{2}, n_{2}\right) & =\int_{0}^{1} \sin ^{2}(\pi n r) \frac{\sin ^{2}\left(\pi n_{2} r\right)}{r^{2}} d r \\
& =\frac{1}{2} \int_{0}^{1} \frac{\sin ^{2}\left(\pi n_{2} r\right)}{r^{2}} d r-\frac{1}{2} \int_{0}^{1} \cos (2 \pi n r) \frac{\sin ^{2}\left(\pi n_{2} r\right)}{r^{2}} d r .
\end{aligned}
$$

The second term in (6.22) is bounded by $O\left(\frac{N_{2}^{4}}{N^{2}}\right)$ for $n>N$, and therefore its contribution to (6.21) is at most

$$
O\left(\frac{N_{2}^{2}}{N^{2}}\right)\left\|\sum_{n_{2} \sim N_{2}}\left(\left|g_{n_{2}}(\omega)\right|^{2}+1\right)\right\|_{L^{q_{1}}(d \omega)}<O\left(\frac{q_{1} N_{2}^{3}}{N^{2}}\right)<O\left(q_{1} N_{2}^{-1}\right)
$$

for $N>N_{2}^{2}$. 
Hence, we may restrict $n$ in (6.21) to the range $n \leq N_{2}^{2}$ and get the bound

$O\left(\log N_{2}\right) \max _{n<N_{2}^{2}}\left\|\sum_{n_{2} \sim N_{2}} \frac{c\left(n, n, n_{2}, n_{2}\right)}{n_{2}^{2}}\left(\left|g_{n_{2}}(\omega)\right|^{2}-1\right)\right\|_{L^{q_{1}}(d \omega)}<O\left(\log N_{2}\right) q_{1} N_{2}^{-\frac{1}{2}}$.

Taking $q_{1} \sim \lambda N_{2}^{\frac{1}{3}}$ and applying Tchebycheff's inequality, (6.20) follows.

Having estimated the contributions of (4.14), (4.15) and (4.17), this completes our analysis of the nonlinear term (4.5).

\section{Further probabilistic CONSIDERATIONS}

Returning to the nonlinear term (4.1), an inspection of the estimates in Section 5 and Section 6 - including Lemma 6.1 and Lemma 6.2 - as well as the nonprobabilistic inequality (4.19) which determines the size of $T$, gives the following statement.

Proposition 7.1. Let $T$ be as in (4.2) and take $M_{i} \leq N_{i}$ for $i=2,3, M=$ $M_{2}+M_{3}$. Moreover, let $u=u_{\phi}$ denote the solution of some truncated equation (1.1). Then

$$
\begin{aligned}
&\left\|\int_{0}^{t} e^{i(t-\tau) \Delta} P_{N}\left[\left(P_{N_{1}} U^{1}\right) \overline{\left(P_{M_{2} \leq n \leq N_{2}} u\right)}\left(P_{M_{3} \leq n \leq N_{3}} u\right)\right](\tau) d t\right\| \\
& \leq 10^{-3}\left\|\mid U^{1}\right\|
\end{aligned}
$$

holds for all $U^{1}$ for which the right side is finite, assuming that $\phi$ is restricted to the complement of an exceptional set of measure at most $\exp \left(-M^{c}\right)$ (with $c>0$ some constant).

Note that for $M$ small, we have the bound (cf. (5.1))

$$
\begin{aligned}
\sup _{\|v\| \leq 1}\left(\int_{B} \int_{0}^{1}\left|P_{N} v \| P_{N_{1}} U^{1}\right|\left|P_{M_{2}} u\right|\left|P_{M_{3}} u\right| d x d t\right) \\
\quad \leq \sup _{\|v\| \leq 1}\left(\|v\|_{L_{t, x}^{2}}\left\|U^{1}\right\|_{L_{t, x}^{2}}\left\|P_{M} u\right\|_{L_{t}^{\infty} L_{x}^{\infty}}^{2}\right) \\
\quad \lesssim T\left\|\left|U^{1}\left\|\mid M^{3}\right\| u \|_{L_{t}^{\infty} L_{x}^{2}}^{2}\right.\right. \\
\quad \leq T M^{3}\|\phi\|_{L_{x}^{2}}^{2}\|\| U^{1} \|,
\end{aligned}
$$

where the second inequality follows from Lemma 2.3 and the third inequality is a consequence of the conservation of the $L_{x}^{2}$ norm under the flow.

Recalling also the discussion in Section 4 on how to treat (4.1) with solutions $u^{(2)}$ and $u^{(3)}$ obtained from different truncations, we obtain

Proposition 7.2. Let $T$ be given by (4.2). Then,

$$
\begin{aligned}
\left\|\int_{0}^{t} e^{i(t-\tau) \Delta} P_{N}\left[\left(P_{N_{1}} U^{1}\right) \overline{\left(P_{N_{2}} u^{(2)}\right)}\left(P_{N_{3}} u^{(3)}\right)\right](\tau) d r \mid\right\| & \\
& \leq 10^{-3}\left\|\left|U^{1}\right|\right\|
\end{aligned}
$$


holds for all $U^{1}$ for which the right side is finite. Here $\left.u^{(i)}\right|_{t=0}=P_{N^{(i)}} \phi$ satisfies the $N^{(i)}$-truncated equation $(i=2,3)$ and we assume $\phi$ is outside an exceptional set of measure at most $O\left(\exp \left(-T^{-c}\right)\right)$ (independent of $\left.U^{1}\right)$.

As we will see in the next section, Proposition 7.2 suffices to establish almost sure convergence of the sequence $\left\{u^{N}\right\}$ of truncated solutions of (1.1), letting $N$ run over the integers $2^{j}$ (or any sufficiently rapidly increasing sequence). However, the measure estimates do not quite suffice to conclude immediately the a.s. convergence of the full sequence, and an additional consideration is needed. The idea is basically the following: in view of Proposition 7.1, we obtain the desired measure estimates for factors $P_{n \geq M_{2}} u^{(2)}$ and $P_{n \geq M_{3}} u^{(3)}$ provided that for instance $M$ satisfies

$$
M=M_{2}+M_{3}>\left(\log \left(N^{(2)}+N^{(3)}\right)\right)^{C}
$$

with $C$ an appropriate constant.

It then remains to consider

$$
\int_{0}^{t} e^{i(t-T) \Delta} P_{N}\left[\left(P_{N_{1}} U^{1}\right) \overline{\left(P_{M} u^{(2)}\right)}\left(P_{M} u^{(3)}\right)\right](\tau) d \tau .
$$

Fix some truncation $M<N^{(0)}<N^{(2)}, N^{(3)}$ and let $u^{(0)}=P_{N^{(0)}} u^{(0)}$ be the corresponding solution of (1.1) with initial data $\left.u^{(0)}\right|_{t=0}=P_{N^{(0)}} \phi$.

We compare (7.4) with

$$
\int_{0}^{t} e^{i(t-\tau) \Delta} P_{N}\left[\left(P_{N_{1}} U^{1}\right) \overline{\left(P_{M} u^{(0)}\right)}\left(P_{M} u^{(0)}\right)\right](\tau) d \tau .
$$

The difference between (7.4) and (7.5) may then be bounded by

$$
\begin{gathered}
\left\|P_{N_{1}} U^{1}\right\|_{L_{t}^{4} L_{x}^{2}}\left[\left\|P_{M} u^{(0)}-P_{M} u^{(2)}\right\|_{L_{t}^{4} L_{x}^{\infty}}+\left\|P_{M} u^{(0)}-P_{M} u^{(3)}\right\|_{L_{t}^{4} L_{x}^{\infty}}\right]\left\|P_{M} u^{(0)}\right\|_{L_{t}^{4} L_{x}^{\infty}} \\
+\left\|P_{N_{1}} U^{1}\right\|_{L_{t}^{4} L_{x}^{2}}\left\|P_{M} u^{(0)}-P_{M} u^{(2)}\right\|_{L_{t}^{4} L_{x}^{\infty}}\left\|P_{M} u^{(0)}-P_{M} u^{(3)}\right\|_{L_{t}^{4} L_{x}^{\infty}} \\
\lesssim\|\| P_{N_{1}} U^{1}\left\|\mid M^{3}\right\| P_{M} \phi \|_{L_{x}^{2}} \\
\cdot\left[\|\| P_{M} u^{(0)}-P_{M} u^{(2)}\left\|\left|+\left\|P_{M} u^{(0)}-P_{M} u^{(3)}\right\|\right|\right]\right. \\
+\left\|P_{N_{1}} U^{1}\left|\left\|M^{3}\right\|\right| P_{M} u^{(0)}-P_{M} u^{(2)}\right\|\|\| P_{M} u^{(0)}-P_{M} u^{(3)}\|\| . \quad(7.6)
\end{gathered}
$$

The interest of this construction is that in order to bound (7.5), only exceptional sets related to $u_{\phi}^{(0)}$ have to be removed, while the prefactor $M^{3}$ in (7.6) is harmless in view of the smallness of $\left\|P_{M} u^{(0)}-P_{M} u^{(i)}\right\| \mid, i=2,3$. This will be made more precise in the next section. 


\section{Proof of the theorem}

In this section, we complete the proof of our main theorem. Toward this end, let $1 \ll N_{0}<N$ be given. Our goal is to compare the solutions $u^{N_{0}}$ and $u^{N}$ of

$$
\left\{\begin{array}{l}
i u_{t}^{N_{0}}+\Delta u^{N_{0}}-P_{N_{0}}\left(u^{N_{0}}\left|u^{N_{0}}\right|^{2}\right)=0 \\
u^{N_{0}}(0)=P_{N_{0}} \phi
\end{array}\right.
$$

and

$$
\left\{\begin{array}{l}
i u_{t}^{N}+\Delta u^{N}-P_{N}\left(u^{N}\left|u^{N}\right|^{2}\right)=0 \\
u^{N}(0)=P_{N} \phi
\end{array}\right.
$$

on a time interval $I=[0, \eta]$ with $\eta>0$ a sufficiently small constant.

Let $1 \ll M \leq N_{0}$ and set

$$
T=\frac{c}{\log M}
$$

with $c>0$ taken as in Proposition 7.2 with $N_{i} \leq M$ for $i=2,3$.

The argument consists of dividing $[0, \eta]$ into time intervals of size $T$ and applying Duhamel's formula on each of these subintervals in order to obtain recursive inequalities.

Taking $0 \leq t \leq T$, we have

$$
u^{N}(t)=e^{i t \Delta}\left(P_{N} \phi\right)+i \int_{0}^{t} e^{i(t-\tau) \Delta} P_{N}\left(u_{N}\left|u^{N}\right|^{2}\right)(\tau) d \tau
$$

and

$$
P_{M}\left(u^{N}-u^{N_{0}}\right)(t)=i \int_{0}^{t} e^{i(t-\tau) \Delta}\left[P_{M}\left(u^{N}\left|u^{N}\right|^{2}\right)-P_{M}\left(u^{N_{0}}\left|u^{N_{0}}\right|^{2}\right) \mid(\tau) d \tau .\right.
$$

We will make an estimate of the $|\|\cdot \mid\|$ norm of this quantity.

We first replace $u^{N}$ and $u^{N_{0}}$ in (8.4) by $P_{M} u^{N}$ and $P_{M} u^{N_{0}}$, respectively. The ||l||| norm of the difference may then be estimated by

$$
\begin{aligned}
{\left[\left\|u^{N_{0}}-P_{M} u^{N_{0}}\right\|_{L_{x}^{3+} L_{t}^{6}}+\left\|u^{N}-P_{M} u^{N}\right\|_{L_{x}^{3+} L_{t}^{6}}\right] } \\
\cdot\left[\left\|u^{N_{0}}\right\|_{L_{x}^{6-} L_{t}^{6}}^{2}+\left\|u^{N}\right\|_{L_{x}^{6-} L_{t}^{6}}^{2}\right]<M^{-\frac{1}{4}},
\end{aligned}
$$

where we have used the a priori bound given by Lemma 3.1, again, 8.5 holds outside an exceptional set of measure at most $O\left(e^{-M^{c}}\right)$.

We then obtain

$$
\begin{aligned}
\left\|P_{M}\left(u^{N}-u^{N_{0}}\right) \mid\right\|<M^{-\frac{1}{4}} & \\
& +\left\|\mid \int_{0}^{t} e^{i(t-\tau) \Delta}\left[P_{M}\left(u^{N}-u^{N_{0}}\right)\left|P_{M} u^{N}\right|^{2}\right](\tau) d \tau\right\| \| \\
+\| & \int_{0}^{t} e^{i(t-\tau) \Delta}\left[\left(P_{M} u^{N_{0}}\right) \overline{\left(P_{M}\left(u^{N}-u^{N_{0}}\right)\right)}\left(P_{M} u^{N}\right)\right](\tau) d \tau\|\|
\end{aligned}
$$




$$
+\||| \int_{0}^{t} e^{i(t-\tau) \Delta}\left[\left|P_{M} u^{N_{0}}\right|^{2}\left(P_{M}\left(u^{N}-u^{N_{0}}\right)\right](\tau) d \tau \mid \| .\right.
$$

In view of Proposition 7.2, each of the terms (8.6), (8.7), (8.8) may be bounded by $10^{-3}\left\|\left|P_{M}\left(u^{N}-u^{N_{0}}\right)\right|\right\|$, provided that $\phi$ is taken outside an exceptional set of measure at most $\exp \left(-T^{-c}\right)$. Note that this set depends on $N_{0}$ and $N$. The preceding discussion then implies that

$$
\|\left.\left|P_{M}\left(u^{N}-u^{N_{0}}\right)\right|\right|_{0, \frac{1}{2} ; T}<2 M^{-\frac{1}{4}}
$$

and an application of Lemma 2.1 gives the existence of some $t_{1} \in\left[\frac{T}{2}, T\right]$ such that

$$
\left\|P_{M}\left(u^{N}-u^{N_{0}}\right)\left(t_{1}\right)\right\|_{L_{x}^{2}}<2 C_{1} M^{-\frac{1}{4}} .
$$

Consider now the next time interval $\left[t_{1}, t_{1}+T\right]$ and write for each $t \in[0, T]$

$$
u^{N}\left(t_{1}+t\right)=e^{i t \Delta}\left(u^{N}\left(t_{1}\right)\right)+i \int_{0}^{t} e^{i(t-\tau) \Delta} P_{N}\left(u^{N}\left|u^{N}\right|^{2}\right)\left(t_{1}+\tau\right) d \tau .
$$

Repeating the above argument, we obtain

$$
\begin{aligned}
\left\|P_{M}\left(u^{N}-u^{N_{0}}\right)\left(t_{1}+\cdot\right) \mid\right\| \leq C_{0}\left\|P_{M}\left(u^{N}-u^{N_{0}}\right)\left(t_{1}\right)\right\|_{L_{x}^{2}}+M^{-\frac{1}{4}} \\
+\frac{3}{10^{3}}\left\|\mid P_{M}\left(u^{N}-u^{N_{0}}\right)\left(t_{1}+\cdot\right)\right\| \|
\end{aligned}
$$

and thus

$$
\|\left|P_{M}\left(u^{N}-u^{N_{0}}\right)\left(t_{1}+\cdot\right)\right|||<2\left(2 C_{0} C_{1}+1\right) M^{-\frac{1}{4}}
$$

for $\phi$ outside a set of measure at most $\exp \left(-T^{-c}\right)$.

Note that the value of $t_{1}$ in (8.10) depends on $\phi$ but this does not create problems with the estimates of the nonlinear terms.

Again by Lemma 2.1, (8.12) gives $t_{2} \in\left[t_{1}+\frac{T}{2}, t_{1}+T\right]$ with

$$
\left\|P_{M}\left(u^{N}-u^{N_{0}}\right)\left(t_{2}\right)\right\|_{L_{x}^{2}}<2 C_{1}\left(C_{0} C_{1}+1\right) M^{-\frac{1}{4}} .
$$

Repeating this argument recursively, we obtain times $t_{j+1} \in\left[t_{j}+\frac{T}{2}, t_{j}+T\right]$ for each $j \geq 1$,with

$$
\left\|P_{M}\left(u^{N}-u^{N_{0}}\right)\left(t_{j+1}\right)\right\|_{L_{x}^{2}} \leq 2 C_{1}\left[C_{0}\left\|P_{M}\left(u^{N}-u_{N_{0}}\right)\left(t_{j}\right)\right\|_{2}+M^{-\frac{1}{4}}\right] .
$$

Iterating the resulting bounds gives

$$
\left\|P_{M}\left(u^{N}-u^{N_{0}}\right)\left(t_{j}\right)\right\|_{L_{x}^{2}}<\left(4 C_{1} C_{0}\right)^{j} M^{-\frac{1}{4}}<M^{-\frac{1}{8}},
$$

since $j \leq T^{-1} \eta=c^{-1} \eta \log M$ by (8.3), and provided that $\eta$ is chosen sufficiently small.

Since

$$
\text { ||| } P_{M}\left(u^{N}-u^{N_{0}}\right)\left(t_{j}+\cdot\right)|| \mid<M^{-\frac{1}{8}}
$$

for each $j$, it follows from Lemma 2.1 that

$$
\frac{1}{T} \int_{I}\left\|P_{M}\left(u^{N}-u^{N_{0}}\right)\right\|_{L_{x}^{2}}^{2} d t \lesssim M^{-\frac{1}{4}}
$$


for each subinterval $I \subset[0, \eta]$ of size $T$. We therefore obtain

$$
\left\|P_{M}\left(u^{N}-u^{N_{0}}\right)\right\|_{L_{t<\eta}^{2} L_{x}^{2}} \lesssim M^{-\frac{1}{8}}
$$

for $\phi$ outside an exceptional set of measure at most $\frac{1}{T} \exp \left(-T^{-c}\right)<\exp \left(-T^{-c^{\prime}}\right)$ (depending on $N_{0}$ and $N$ ).

In view of the apriori bounds of Proposition 3.2 on the quantities $\left\|u^{N_{0}}\right\|_{X^{s, b}}$ and $\left\|u^{N}\right\|_{X^{s, b}}$ for $s<\frac{1}{2}$ and $b<\frac{3}{4}$ ) and interpolation arguments, the bound (8.16) also implies

$$
\left\|u^{N}-u^{N_{0}}\right\|_{X^{s, b}[0, \eta]}<M^{-c(s, b)}
$$

for $s<\frac{1}{2}$ and $b<\frac{3}{4}$.

To consider the interval $[\eta, 2 \eta]$, we repeat the previous reasoning with $M$ replaced by $M_{1}=M^{c}$ and $T$ by $T_{1}=\frac{c}{\log M_{1}}$. This gives

$$
\left\|u^{N}-u^{N_{0}}\right\|_{X^{s, b}[\eta, 2 \eta]}<M_{1}^{-c(s, b)}
$$

and so on.

Starting from $M=N_{0}$, the above argument shows that for any given time interval $[0, T]=I$ with $T<\infty$, the estimate

$$
\left\|u^{N}-u^{N_{0}}\right\|_{X^{s, b}(I)}<N_{0}^{-c(s, b, T)}
$$

holds for $s<\frac{1}{2}, b<\frac{3}{4}$ and all $\phi$ outside a set of measure at most $e^{-\left(\log N_{0}\right)^{c}}$, depending on $N$ and $N_{0}$. This statement clearly implies convergence of the sequence $\left\{u^{N}\right\}, N=2^{j}$ in

$$
\bigcap_{s<\frac{1}{2}, b<\frac{3}{4}} X^{s, b}(I)
$$

almost surely in $\phi$.

Since the series

$$
\sum_{N \in \mathbb{Z}_{+}} e^{-(\log N)^{c}}
$$

diverges, this does not immediately imply the convergence of the full sequence. In order to achieve this improvement of the convergence properties, we use the procedure discussed at the end of Section 7 .

Toward this end, fix $N_{0} \gg 1$ and let $N$ range between $N_{0}$ and $2 N_{0}$. In (7.5), let $u^{(0)}=u^{N_{0}}$, and take $M$ as the truncation

$$
K=\left(\log N_{0}\right)^{C}
$$

with $C$ a sufficiently large constant.

On the other hand, in the inequality (8.6) $-(8.8)$ above, $\log M \sim \log N_{0}$. Recalling (7.6), the estimation of (8.6)-(8.8) gives some additional terms:

$$
\begin{aligned}
&\left\|\left|P_{M}\left(u^{N}-u^{N_{0}}\right)\right|\right\| \\
&<M^{-\frac{1}{4}}+10^{-3}\left\|\left|P_{M}\left(u^{N}-u^{N_{0}}\right)\right|\right\|
\end{aligned}
$$




$$
\begin{aligned}
& +K^{3}\left\|P_{K} \phi\right\|_{L_{x}^{2}}\left\|P_{K}\left(u^{N}-u^{N_{0}}\right)\right\|||\left\|P_{M}\left(u^{N}-u^{N_{0}}\right)\right\| \mid \\
& +K^{3}\left|\left\|P_{K}\left(u^{N}-u^{N_{0}}\right)\right\|\right|^{2}\left\|P_{M}\left(u^{N}-u^{N_{0}}\right)\right\| \mid \\
<M^{-\frac{1}{4}} & +\left[10^{-3}+K^{3}\|\phi\|_{L_{x}^{2}}\left|\left\|P_{M}\left(u^{N}-u^{N_{0}}\right)\right\|\right|\right. \\
& +K^{3}\left|\left\|P_{M}\left(u^{N}-u^{N_{0}}\right)|\||^{2}\right] \cdot\right||| P_{M}\left(u^{N}-u^{N_{0}}\right) \| \mid
\end{aligned}
$$

The inequality (8.20) holds for $\phi$ outside an exceptional set which is the union of a set of measure at most $e^{-\left(\log N_{0}\right)^{c}}$ depending on $N_{0}$ and an exceptional set of measure at most $e^{-K^{c}}<N_{0}^{-2}$ depending on $N$.

Taking $\|\phi\|_{L_{x}^{2}}<K$ in (8.20) and recalling that $\log M \sim \log N_{0}$, we may again conclude (8.9), which is now valid for all $N_{0} \leq N \leq 2 N_{0}$ and $\phi$ outside an exceptional set of measure at most $e^{-\left(\log N_{0}\right)^{c}}$.

This completes the proof of the main theorem.

\section{REFERENCES}

[1] J. Bourgain. Fourier transform restriction phenomena for certain lattice subsets and applications to nonlinear evolution equations. I. Schrödinger equations. Geom. Funct. Anal. 3 (1993), no. 2, 107-156.

[2] J. Bourgain. Fourier transform restriction phenomena for certain lattice subsets and applications to nonlinear evolution equations. II. The KdV-equation. Geom. Funct. Anal. 3 (1993), no. 3, 209-262.

[3] J. Bourgain. Periodic nonlinear Schrödinger equation in invariant measures. Comm. Math. Phys. 166 (1994), 1-24.

[4] J. Bourgain. On the Cauchy and invariant measure problem for the periodic Zakharov system. Duke Math. J. 76 (1994), no. 1, 175202.

[5] J. Bourgain. Invariant measures for the 2D-defocusing nonlinear Schrödinger equation. Comm. Math. Phys. 176 (1996), 421-445.

[6] J. Bourgain. Invariant measures for NLS in infinite volume. Comm. Math. Phys. 210 (2000), no. 3, 605620 .

[7] J. Bourgain. Nonlinear Schödinger equations. Hyperbolic equations and frequency interactions (Park City, UT, 1995), 3157, IAS/Park City Math. Ser., 5, Amer. Math. Soc., Providence, RI, 1999.

[8] J. Bourgain and A. Bulut. Gibbs measure evolution in radial nonlinear wave and Schrödinger equations on the ball. Comptes Rendus Math. 350 (2012) 11-12, pp. 571-575.

[9] J. Bourgain and A. Bulut. Invariant Gibbs measure evolution for the radial nonlinear wave equation on the three dimensional ball. Preprint. (2012)

[10] J. Bourgain and A. Bulut. Almost sure global well posedness for radial NLS on the unit ball I: the 2D case. Preprint. (2012)

[11] N. Burq and N. Tzvetkov. Random data Cauchy theory for supercritical wave equations. I. Local theory and II. A global existence result. Invent. Math. 173 (2008), no. 3, 449-475 and 477-496. 
(J. Bourgain) Institute for Advanced Study, Princeton, NJ 08540

E-mail address: bourgain@math.ias.edu

(A.Bulut) Institute for Advanced Study, Princeton, NJ 08540

E-mail address: abulut@math.ias.edu 